c. 2

Army Corps Engineers

... uterways Experiment

Station

\title{
Instream Flow Incremental Methodology: A Synopsis with Recommendations for Use and Suggestions for Future Research
}

\author{
by John M. Nestler \\ Environmental Laboratory
}

Approved For Public Release; Distribution Is Unlimited

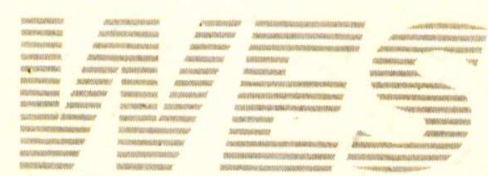

RESEARCH LIBRARY

US ARMY ENGINEER WATERWAYS

EXPERIMENT STATION

VICKSBURG, MISSISSIPPI 
The contents of this report are not to be used for advertising, publication, or promotional purposes. Citation of trade names does not constitute an official endorsement or approval of the use of such commercial products.

PRINTED ON RECYCLED PAPER 


\title{
Instream Flow Incremental Methodology: A Synopsis with Recommendations for Use and Suggestions for Future Research
}

\author{
by John M. Nestler \\ Environmental Laboratory \\ U.S. Army Corps of Engineers \\ Waterways Experiment Station \\ 3909 Halls Ferry Road \\ Vicksburg, MS 39180-6199
}

Final report

Approved for public release; distribution is unlimited 


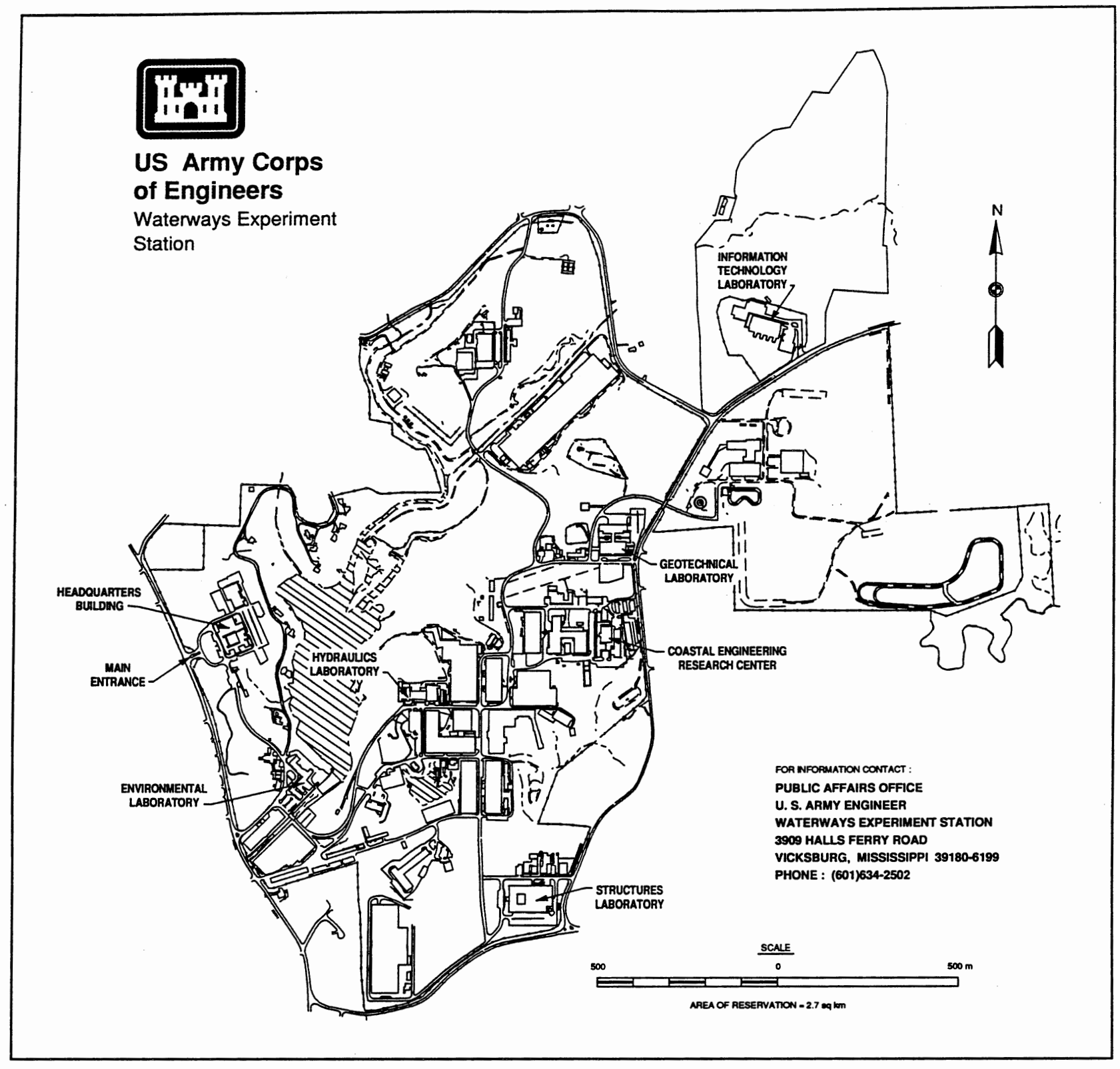

\section{Waterways Experiment Station Cataloging-in-Publication Data}

Nestler, John M.

Instream Flow Incremental Methodology : a synopsis with recommendations for use and suggestions for future research / by John M. Nestler ; prepared for U.S. Army Corps of Engineers.

48 p. : ill. ; $28 \mathrm{~cm}$. - (Technical report ; EL-93-3) Includes bibliographical references.

1. Water resources development - Environmental aspects - United States. 2. River engineering - United States - Environmental aspects. 3. Stream ecology. 4. Hydrology - United States. I. United States. Army. Corps of Engineers. II. U.S. Army Engineer Waterways Experiment Station. III. Environmental Impact Research Program (U.S.) IV. Title. V. Series: Technical report (U.S. Army Engineer Waterways Experiment Station) ; EL-93-3.

TA7 W34 no.EL-93-3 
This report was prepared by the Environmental Laboratory (EL), US Army Engineer Waterways Experiment Station (WES), Vicksburg, MS, and forms part of the Environmental Impact Research Program (EIRP), Work Unit 32494, "Assessing Effects of Reservoir Operation on Downstream Aquatic Resources." The EIRP is sponsored by Headquarters, US Army Corps of Engineers (HQUSACE), and is assigned to WES under the management of the EL. Dr. Roger T. Saucier, EL, is the Program Manager for the EIRP. The HQUSACE Technical Monitors for EIRP are Dr. John Bushman, Mr. Pete Juhle, and Mr. Dave Mathis.

This report was written by Dr. John M. Nestler, Water Quality and Contaminant Modeling Group (WQCMG), EL, under the general supervision of Dr. Mark S. Dortch, Chief, WQCMG; Mr. Donald L. Robey, Chief, Environmental Processes and Effects Division, EL; and Dr. John Harrison, Director, EL. In-house technical review was performed by Ms. L. Toni Schneider and Mr. Tom Cole, WQCMG. Outside technical review was provided by Dr. Robert Milhous of the National Ecology Research Center, US Fish and Wildlife Service. Ms. Laura Scott, WQCMG, prepared graphics for this report.

At the time of publication of this report, Director of WES was Dr. Robert W. Whalin. Commander was COL Leonard G. Hasse11, EN.

This report should be cited as follows:

Nestler, John M. 1993. "Instream Flow Incremental Methodology: A Synopsis with Recommendations for Use and Suggestions for Future Research," Technical Report EL-93-3, US Army Engineer Waterways Experiment Station, Vicksburg, MS . 
PREFACE . . . . . . . . . . . . . . . . . . . . . . . . . . . . 1

LIST OF TABLES . . . . . . . . . . . . . . . . . . . . . . . . . 3

LIST OF FIGURES . . . . . . . . . . . . . . . . . . . . . . . . . . . 3

PART I : INTRODUCTION . . . . . . . . . . . . . . . . . . . . . . . . 4

PART II: IFIM AND WATER RESOURCES DEVELOPMENT . . . . . . . . . . . . 6

PART III: PHYSICAL VARIABLES AND REGIONAL CONSIDERATIONS . . . . . . . 8

Hydrologic Methods . . . . . . . . . . . . . . . . . . . . 9

IFIM and Western Hydrology . . . . . . . . . . . . . . . . . . . 13

IFIM and Hydrology in Other Regions of the United States . . . . 22

IFIM and Channel Characteristics . . . . . . . . . . . . . . . . 25

IFIM and Western Mountain Stream Region Applications . . . . . . 25

IFIM in Other Stream Regions . . . . . . . . . . . . . . . . . . 27

PART IV: BIOLOGICAL AND LIFE HISTORY CONSIDERATIONS . . . . . . . . . 29

PART V: DISCUSSION, RECOMMENDATIONS, AND CONCLUSIONS . . . . . . . . 31

Discussion . . . . . . . . . . . . . . . . . . . . . . . . . . 31

Recommendations . . . . . . . . . . . . . . . . . . . 33

Conclusions . . . . . . . . . . . . . . . . . . . . 35

REFERENCES . . . . . . . . . . . . . . . . . . . . . . . . 38 


\section{LIST OF TABLES}

No.

Page

1 Hydrologic Summaries for Select Streams Presented in Figures 1 and 2 Ordered by Basin Size . . . . . . . . . . . . . 14

2 Summary of Hydrologic Trends for Table 1 Streams by Stream Region... . . . . . . . . . . . . . . . . 20

\section{LIST OF FIGURES}

No.

Page

1

Streamflow summaries for streams in the western United States. .

2 Streamflow summaries for streams in the southeastern and eastern United States . . . . . . . . . . . . . . . . . . . . . 11

3 Contrasting flow summaries for western and central United

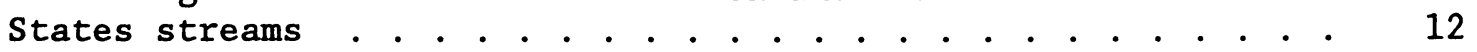

4 Comparison of existing and recommended approaches for relating project impacts on aquatic biota . . . . . . . . . . . 32 
INSTREAM FLOW INCREMENTAL METHODOLOGY: A

SYNOPSIS WITH RECOMMENDATIONS FOR USE

AND SUGGESTIONS FOR FUTURE RESEARCH

PART I: INTRODUCTION

1. Population growth and continued economic development are partially founded on continued development of water resources for power production, water supply, flood control, navigation, and other benefits. Streams and rivers of many regions of the United States are viewed as a preferred source of water supply to promote economic development and support population growth (Petts 1984). Unmanaged stream regulation and diversion have the potential to severely impact environmental quality and fish and wildlife resources. Increased water resources development has intensified the conflict between economic benefits of stream diversion and regulation and the need to protect and maintain the integrity of lotic ecosystems (Petts 1984).

2. Impact assessment methodologies that are quantifiable, repeatable, accepted, and defensible are required to mediate conflicts between water resources development and natural resource preservation (Hobbs, Stakhiv, and Grayman 1989). Such methodologies allow regulators, resource agencies, developers, and development agencies to determine relative impacts of different water resources development plans. Regulators and resource agencies can then identify and support plans that allow for economic development, but minimally impact fish and wildlife resources. Developers can pursue reasonable water resources plans with diminished threat of prolonged studies and litigation, while unacceptable plans can be dropped early in project planning.

3. The most commonly employed means of assessing the impacts of stream regulation and water diversion on aquatic biota is the US Fish and Wildife Service's Instream Flow Incremental Methodology (IFIM) (Reiser, Wesche, and Estes 1989). Although subject to criticism (Patten 1979; Orth and Maughan 1982; Mathur et al. 1984), IFIM is generally accepted as a means of assessing the effects of stream regulation and water diversion on coldwater aquatic biota. However, applications of IFIM in eastern and southeastern United States streams have generally met with scientific skepticism (Bain and Boltz 1989). The present lack of any viable alternatives to IFIM has diminished the ability of resource, regulatory, and development agencies to assess and manage 
the effects of stream regulation and diversion in the eastern and southeastern United States.

4. IFIM mirrors the concepts familiar to ecological modelers that specialize in impact assessment in that models are used to predict environmental impacts of project alternatives on key variables and thus facilitate trade-off analysis between environmental and economic impacts. The most frequently used program library for executing the IFIM is the Physical Habitat Simulation (PHABSIM) system, and it is this system that is also the topic of most reviews and discussions. A variety of options and pathways that vary considerably in their treatment of suitability information and of hydraulic simulation is available within the PHABSIM system (Milhous, Wegner, and Waddle 1981; Milhous, Updike, and Schneider 1989). While some workers have employed innovative applications of IFIM to solve site-specific instream flow problems, most applications of the PHABSIM system use cell-by-cell hydraulic conditions (usually depth, mean cell velocity, and substrate/cover) coupled to lifestage-specific suitability information (see Bovee (1986) for methods) to generate relationships between discharge and habitat. The latter, common approach is the focus of this synthesis paper. For additional background information, IFIM is described in detail in Bovee (1982), and an overview of the methodology with selected examples is presented in Nestler, Milhous, and Layzer (1989).

5. A number of considerations for applying IFIM to stream ecosystems in different regions of the United States are identified herein. For clarity and brevity, these considerations are presented by first briefly describing the relationship of the IFIM to western United States stream regulation/diversion issues. Second, important regional differences in hydrologic patterns and channel geomorphology that may affect application of IFIM are identified. In particular, the interrelated geomorphology and hydrology of western streams are contrasted, concentrating on those streams that led to the early development of IFIM, with hydrologic patterns in the central, eastern, and southeastern United States. Third, the biological significance and implications of these factors for conducting stream assessments are discussed. 
PART II: IFIM AND WATER RESOURCES DEVELOPMENT

6. In the arid West, stream water was used to foster economic development by supporting farming, mining, and population growth (Dauber 1976) until it became increasingly apparent that the public interest for preserving trout and salmon was negatively impacted (Bayha 1976). The early history of IFIM is tightly linked to institutional and legal factors associated with the allocation of water at the state level and with reservoir operation in the Western States (Allred 1976; Caulfield 1976; Bradley 1976). Impetus to develop IFIM as a standard methodology came primarily from state resource agencies and water management agencies seeking to mediate between conflicting uses of stream water and to allocate water consistent with the public interest. Specifically, IFIM can be traced to the following: (a) efforts by state fisheries and water agencies (particularly in Oregon, Washington, and California; e.g., see reviews in Stalnaker and Arnette (1976a)); (b) Federal agencies assisting the efforts of the states (e.g., assistance provided by the Environmental Protection Agency (Bovee, Gore, and Silverman 1978) and the US Geological Survey (e.g., Collings (1972), Collings and Hill (1973))); and (c) Federal agencies having themselves stewardship responsibilities for streamflow (e.g., US Forestry Service (Herrington and Dunham 1967), see historical description in Carlson (1976)). In recognition of the diversity and abundance of this early work, the first mandate of the US Fish and Wildlife Service's Cooperative Instream Flow Service Group was not so much to develop new technologies, but to cooperatively improve and standardize methods based on the efforts of these early workers.* Substantial contributions by national laboratories or academic institutions were notably absent from the early history of IFIM.

7. The plexus of IFIM--hydrology-based assessment, appropriation water law, historical agency associations, and supportive institutional infrastructure--was lost when IFIM was exported to regions outside the arid West. As a consequence, stream biologists in regions outside the arid West viewed the IFIM as foreign, unnecessarily complicated, and out of the mainstream of stream impact assessment methods (Bain and Boltz 1989). The heavy reliance of IFIM on hydrology, a boon to stream biologists in Western States, became a liability in regions in which professionals in resource agencies had

* Personal Communication, Dr. R. Milhous, National Ecology Research Center, Fort Collins, CO. 
less appreciation for the role of hydrologic methods in water resources management and impact assessment. 
PART III: PHYSICAL VARIABLES AND REGIONAL CONSIDERATIONS

8. The following paragraphs describe how the subtle interplay of channel geomorphology, hydrology, and channel size influenced many of the basic tenets of IFIM. Understanding these interrelationships is an important step required to extend application of the IFIM outside of the region in which it was developed. A review of the development of IFIM and early IFIM applications (see Stalnaker and Arnette (1976b), Loar and Sale (1981), and Morhardt (1987) for recent reviews) indicates a general, but not exclusive, geographic association with the Western Mountain Stream Region, one of seven stream regions of the United States identified by Brussock, Brown, and Dixon (1985). Work by Collings (1972, 1974), Collings, Smith, and Higgins (1972a, 1972b), and Collings and Hill (1973) was all performed in western Washington. Work by Dunham and Collotzi (1974) was performed in Utah; and work by Waters (1976) and Kelley, Cordone, and Delisle (1960) was performed in northern California. One of the most comprehensive early reports prepared by the then fledgling Instream Flow Group (Pruitt and Nadeau 1978) featured a number of Idaho trout streams; work documented in Sams and Pearson (1963), Munther (1975), and Thompson (1972) was performed in the Western Mountain Region.

9. General channel characteristics of streams and rivers reflect the long-term effects of regional lithology, landform, hydrology, climate, and resultant vegetation patterns (Beschta and Platts 1986; Garbrecht and Shen 1988; see also discussion by Statzner and Higler (1985)). As Beschta and Platts (1986) further points out, these factors determine stream characteristics such as drainage density, stream order, and longitudinal channel profile. All of these factors ultimately influence regional patterns in nutrient dynamics, riparian vegetation, and instream habitat conditions because they exhibit different trends and different intercorrelations in different stream regions of the United States (Miller and Onesti 1988). Aquatic biota respond to these regional influences. Bowlby and Roff (1986) points out that different habitat factors can limit trout production in streams from different areas of North America. Indeed, Lanka and Hubert (1987) provides evidence that basin geomorphology and stream habitat quality are linked, even within one major stream region drainage. Regional hydrologic patterns exert a profound effect on the structure of biotic communities (Minshall et al. 1983). The importance of particulate organic matter ( $P O M$ ) dynamics in determining the trophic structure of stream communities is well known (Vannote et al. 1980). Hydrologic 
patterns directly determine rates of POM transport (Sedell et al. 1978), import and storage of POM (Gurtz et al. 1988), and indirectly determine stream periphyton growth through an interplay between current velocity and nutrient concentrations (Horner and Welch 1981).

\section{Hydrologic Methods}

10. Based on extensive analyses of regional hydrologic patterns, Poff and Ward (1989) argues that degree of intermittency, flood frequency, flood predictability, and overall flow predictability/variability determine the physical template upon which aquatic communities are structured. Conceptually, similar analyses with generally similar conclusions were performed by Hughes and James (1989) for Australian streams. Harmonic analysis of logtransformed mean monthly flow data from gages in different parts of the United States (sites were obtained from US Geological Survey (1988)) provides further insight into regional hydrologic patterns identified by Poff and Ward (1989). Log-transformed data are more meaningful surrogates of habitat quality than untransformed data since both water depth and water velocity in streams are usually linearly related to the logarithm of stream discharge. Analyses were based on a period of record from 1951 through 1980 to allow direct comparison to stream gaging records as commonly displayed "National Water Conditions" published by the US Geological Survey. Three hydrologic summaries (presented for each stream in Figures 1 and 2) were evaluated by fitting them to a cosine function employing nonlinear regression (PROC NLIN--SAS Institute Inc. (1988)) using

$$
\operatorname{LMEANQ}_{(1)}=\operatorname{AMP} \times(\operatorname{COS}(\text { MONTH }+ \text { PHS })) \times \text { PER X } \pi
$$

where:

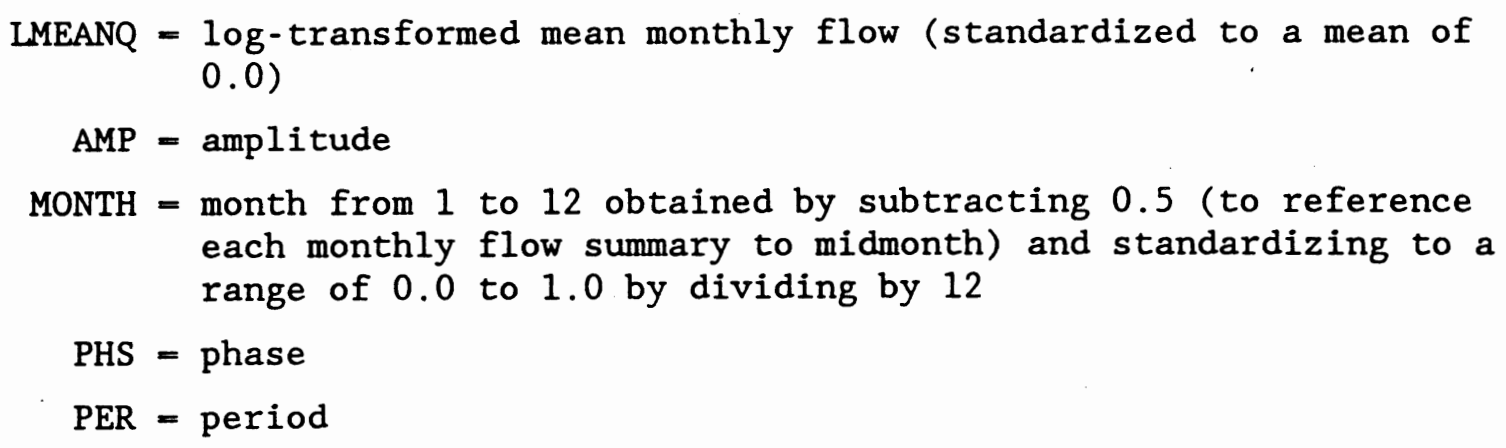
0.0 ) 

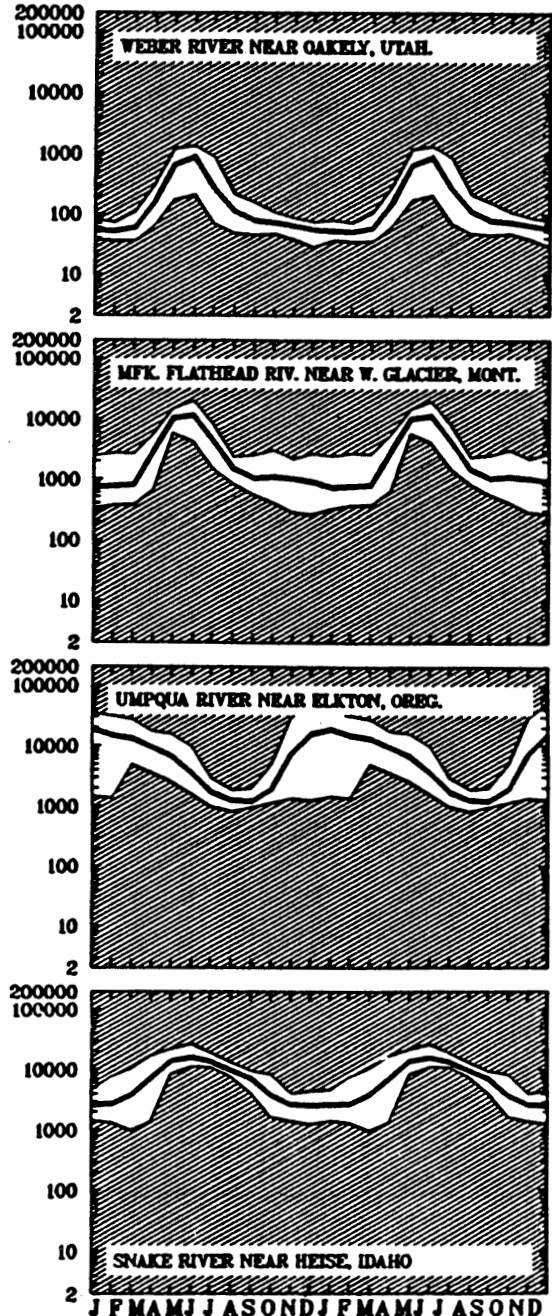
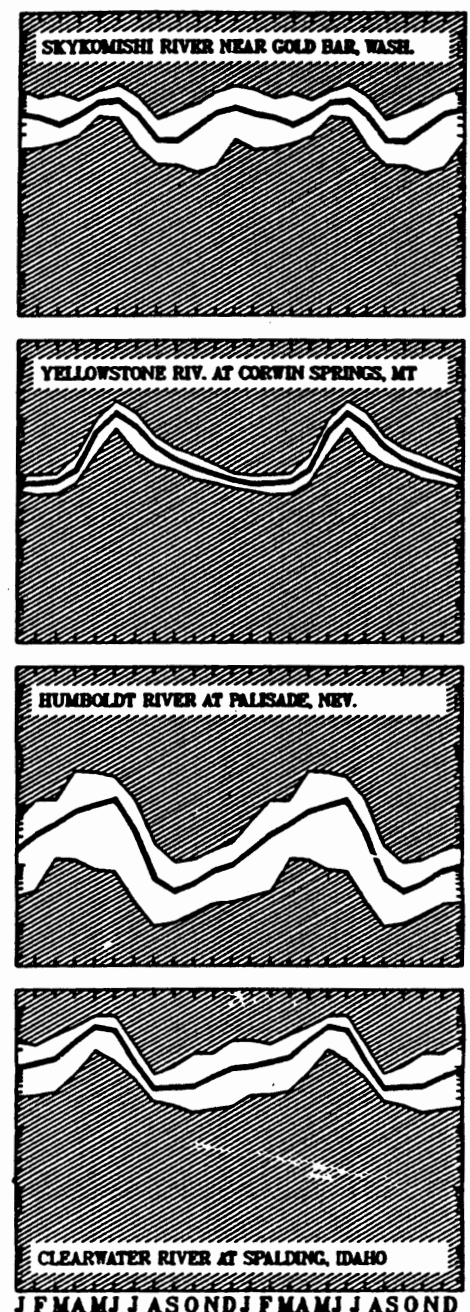
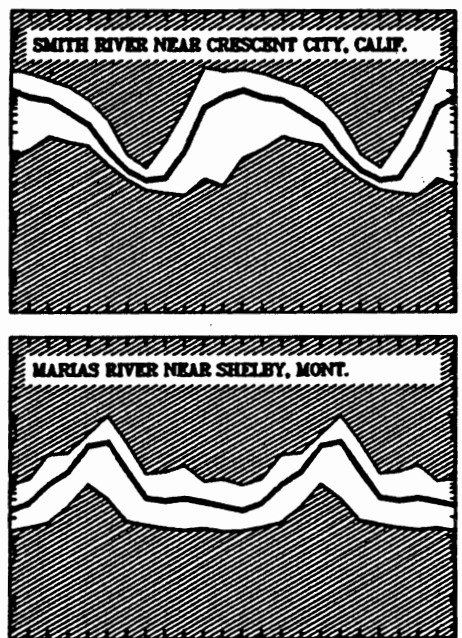

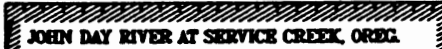
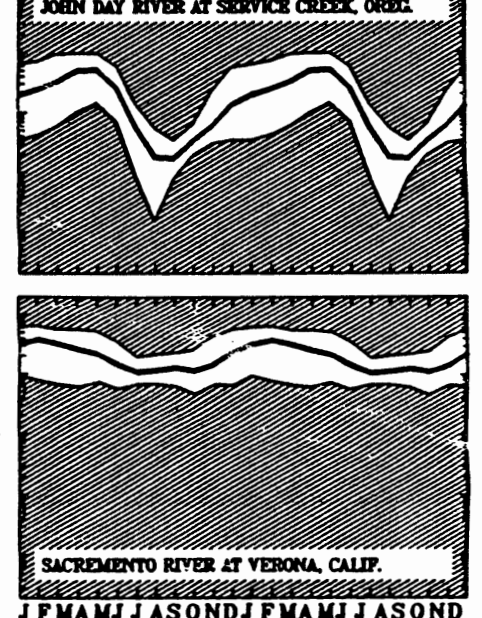

Figure 1. Streamflow summaries for streams in the western United States. The dark lines represent mean monthly flows, and the gray boundaries represent upper and lower ranges in monthly flow for the 1951-1980 reference period. These graphs are reproduced directly from US Geological Survey (1988) with no changes, additions, or deletions. The graphs are reordered in ascending order of drainage basin area for ease of comparison 

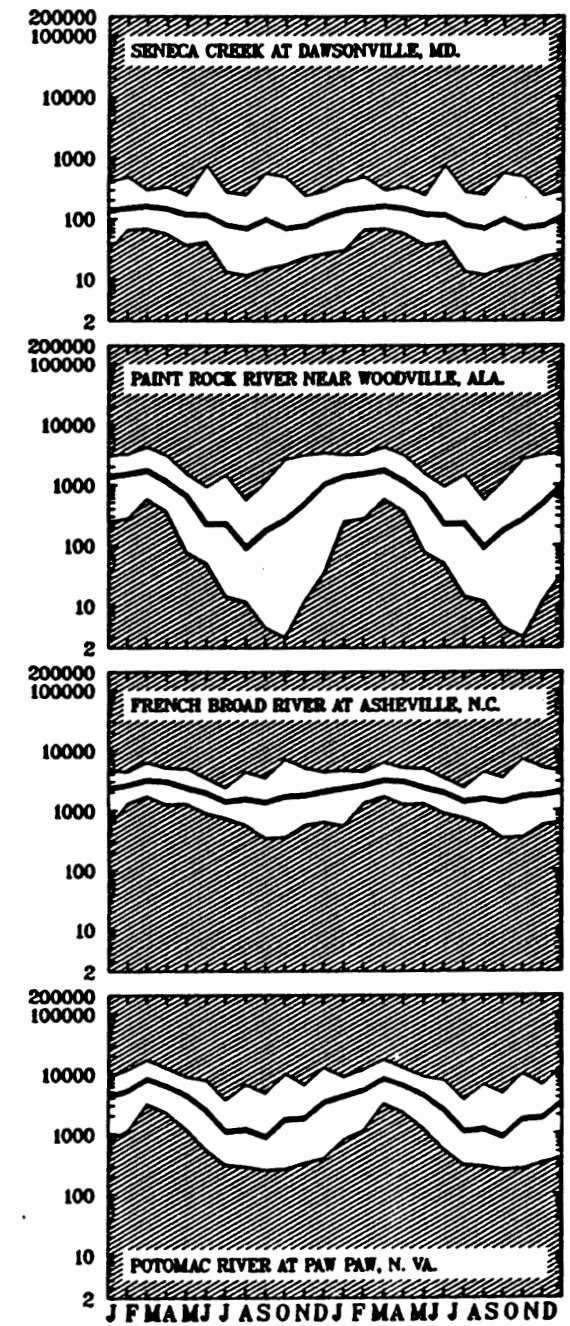
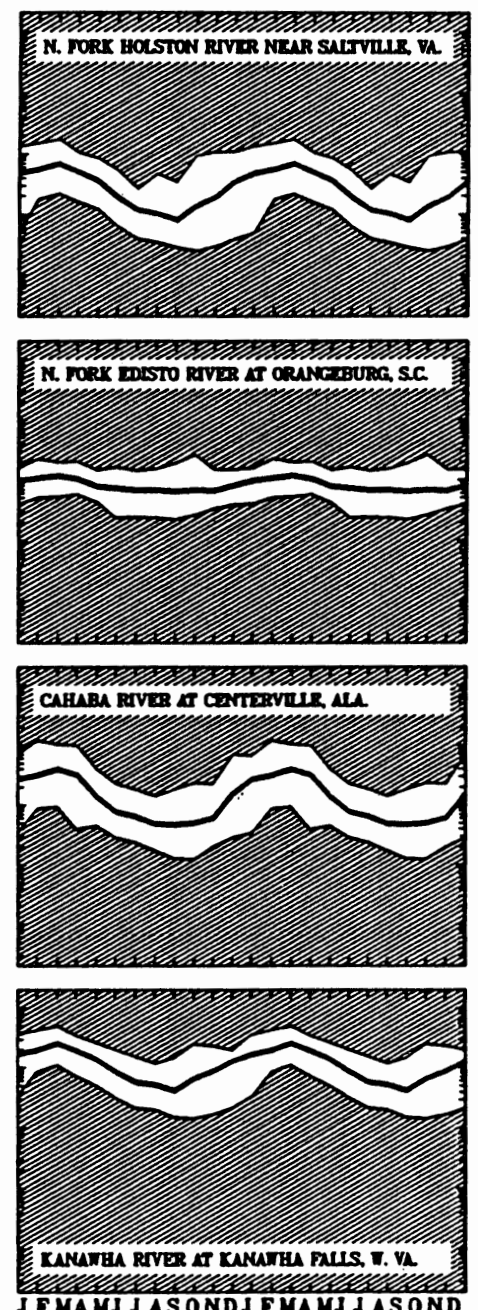
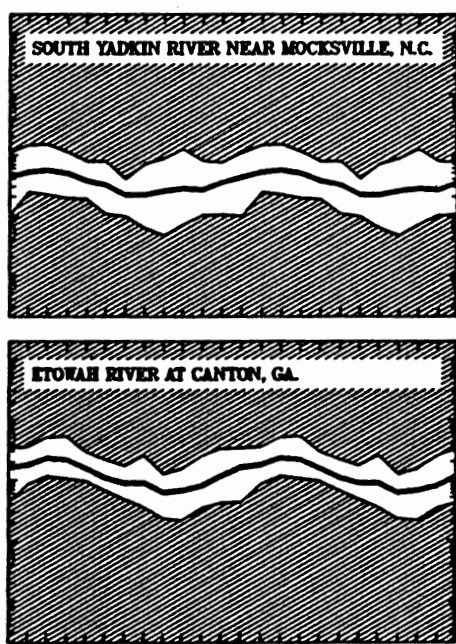

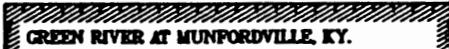
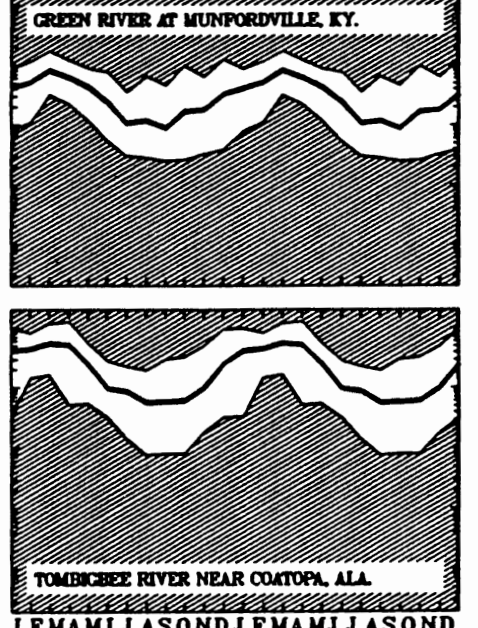

Figure 2. Streamflow summaries for streams in the southeastern and eastern United States (US Geological Survey 1988). Formats and conventions are similar to Figure 1 
11. The 24 sites selected for analysis (Figures 1 and 2 ) were obtained from regional flow summaries published by the US Geological Survey (1988), and an additional two sites (Figure 3 ) were selected to contrast regional streamflow patterns. For each site, harmonic analysis provides information on flow range (AMP), timing of flow pattern (PHS), and insight into underlying hydrologic factors (e.g., winter rain, snowmelt, synoptic flow patterns) that

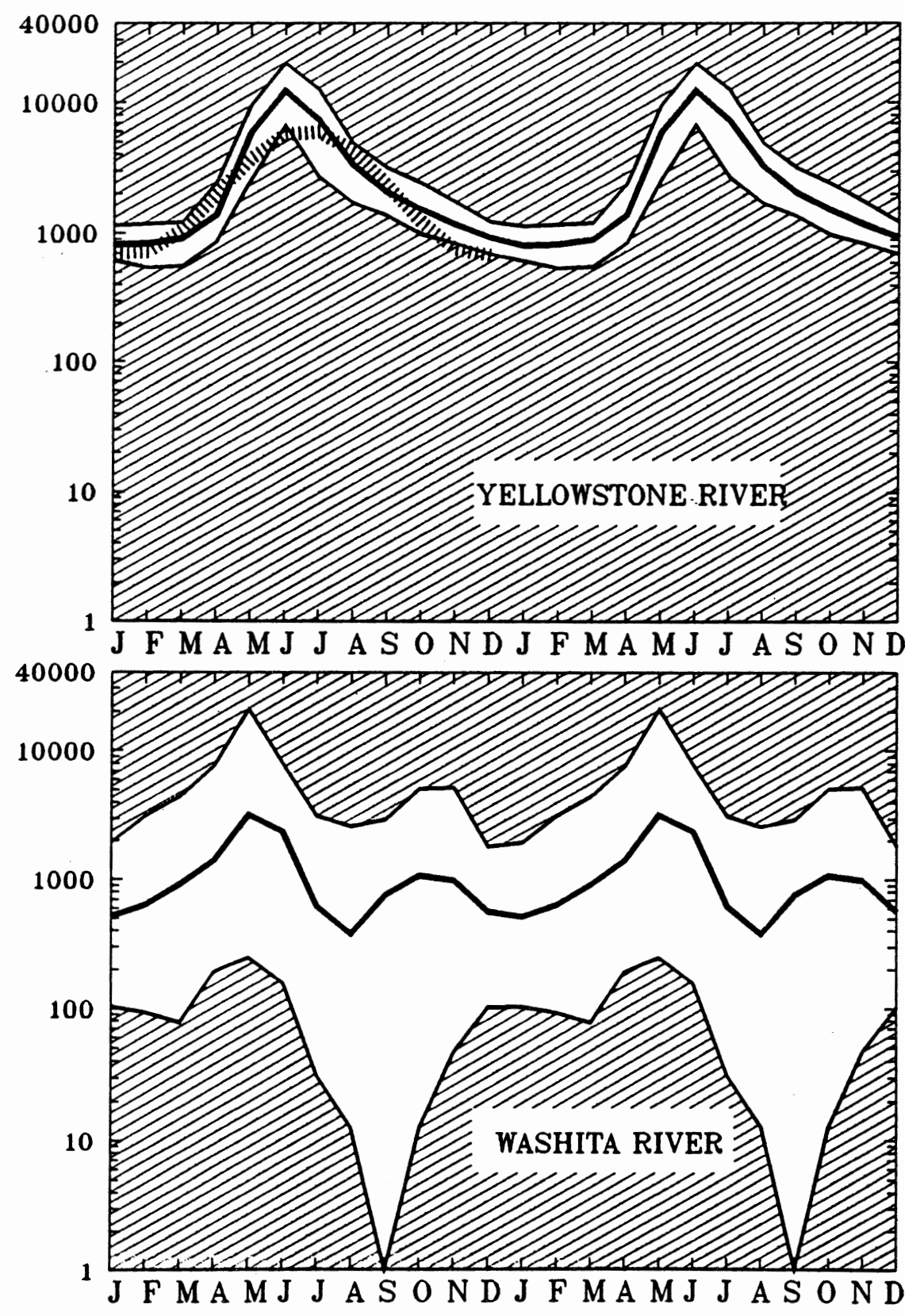

Figure 3. Contrasting flow summaries for western and central United States streams. Note the wellbehaved hydrologic pattern exhibited by the Yellowstone River contrasted to the poorly defined hydrologic pattern of the Washita River 
determine flow pattern (PER). Goodness-of-fit analyses using standard F-tests provide estimates of predictability with highly significant fits suggesting high degrees of hydrologic predictability. While not strictly valid for nonlinear models, both Draper and Smith (1986) and Kimura (1980) suggest using F-tests to approximately assess the fit of nonlinear models. Correlation analysis of the three hydrologic summaries for each stream provides insight into how extended (monthly) periods of high or low flows relate to seasonal hydrologic patterns as depicted by mean monthly flows for the period of record. High correlations between flow summaries for a single site suggest that wet and dry periods tend to fall within the hydrologic pattern depicted by the mean monthly flow summary, whereas low correlations suggest that extended extreme flow conditions occur either randomly during any time of the year or follow a pattern different from mean monthly flows. A "seasonality index" computed as

Mean of Monthly Flows

Associated Amplitude of Monthly Flows

is used to summarize the strength of seasonal pattern for mean monthly flows, minimum monthly flows, and maximum monthly flows. High values of this ratio indicate that flows occur randomly, whereas low values indicate that flows fall within distinct seasonal patterns (assuming an underlying harmonic pattern resembling a cosine function).

\section{IFIM and Western Hydrology}

12. Streams in the Western Mountain Stream Region are dominated by blends of meteorological and climatological characteristics descriptively termed winter rain, snowmelt, and snow and rain (Poff and Ward 1989). In general, these streams are characterized by a relatively high degree of seasonality in monthly flow patterns. Note the generally high correlation coefficients between the mean, minimum, and maximum monthly flows for streams in the Western Mountain Region, particularly for streams that, based on their geographic location, probably receive considerable snowmelt (Table 1). Compared with streams in other regions of the United States, the streams in the Western Mountain Stream Region have higher predictability in both the timing 
Table 1

Hydrologic Summaries for Select Streams Presented in

Figures 1 and 2 Ordered by Basin Size

\begin{tabular}{|c|c|c|c|c|c|c|c|c|c|c|}
\hline \multirow{2}{*}{$\begin{array}{c}\text { Region } \\
\text { and } \\
\text { Status }\end{array}$} & \multirow{2}{*}{$\begin{array}{c}\text { Stream } \\
\text { Name/ } \\
\text { Location } \\
\end{array}$} & \multirow{2}{*}{$\begin{array}{l}\text { Flow } \\
\text { Summary }\end{array}$} & \multirow[b]{2}{*}{ Phase } & \multirow[b]{2}{*}{ Amplitude } & \multirow[b]{2}{*}{ Period } & \multicolumn{2}{|c|}{$\begin{array}{c}\text { Harmonic } \\
\text { Equation Fit }\end{array}$} & \multicolumn{2}{|c|}{$\begin{array}{l}\text { Correlation Summary } \\
\text { Statistics }\left(r^{2} / p\right)\end{array}$} & \multirow{2}{*}{$\begin{array}{c}\text { Log } \\
\text { Annual } \\
\text { Mean } \\
\end{array}$} \\
\hline & & & & & & $p$ & $\mathrm{r}^{2}$ & Min & Max & \\
\hline $\begin{array}{l}\text { ECST } \\
\text { NR } \\
262 \quad \mathrm{KM}\end{array}$ & $\begin{array}{l}\text { Seneca } \\
\text { Creek, } \\
\text { MD }\end{array}$ & $\begin{array}{l}\text { mean } \\
\text { min } \\
\max \end{array}$ & $\begin{array}{r}-0.19 \\
-0.22 \\
0.12\end{array}$ & $\begin{array}{r}0.16 \\
0.35 \\
-0.02\end{array}$ & $\begin{array}{l}1.88 \\
2.13 \\
2.00\end{array}$ & $\begin{array}{l}<0.001 \\
<0.001 \\
>0.75\end{array}$ & $\begin{array}{l}0.86 \\
0.88 \\
0.00\end{array}$ & $0.90 / 0.0001$ & $\begin{array}{l}0.20 / 0.5442 \\
0.16 / 0.6253\end{array}$ & $\begin{array}{l}2.021 \\
1.451 \\
2.553\end{array}$ \\
\hline $\begin{array}{l}\text { ECST } \\
\text { NR } \\
1770\end{array}$ & $\begin{array}{l}\text { N. Fork } \\
\text { Edisto } \\
\text { R., SC }\end{array}$ & $\begin{array}{l}\text { mean } \\
\text { min } \\
\max \end{array}$ & $\begin{array}{l}-0.15 \\
-0.20 \\
-0.04\end{array}$ & $\begin{array}{l}0.12 \\
0.20 \\
0.00\end{array}$ & $\begin{array}{l}1.85 \\
2.20 \\
2.00\end{array}$ & $\begin{array}{l}<0.001 \\
<0.001 \\
>0.75\end{array}$ & $\begin{array}{l}0.90 \\
0.91 \\
0.05\end{array}$ & $0.89 / 0.0001$ & $\begin{array}{l}0.32 / 0.3116 \\
0.20 / 0.5392\end{array}$ & $\begin{array}{l}2.906 \\
2.536 \\
3.239\end{array}$ \\
\hline & & & & & $\ln$ & & & & & \\
\hline
\end{tabular}

Note: Variables defined as follows: region--stream region where ECST = Eastern Coastal Stream Region, EMNT - Eastern Mountain Stream Region, MCONT - Midcontinental Stream Region, EPH - Ephemeral Stream Region, WMNT - Western Mountain Stream Region; status--regulation status where NR - not regulated, $S R$ = slightly regulated, $P R$ = partly regulated, and $R$ = regulated; flow summary--mean refers to mean monthly ( $\log )$ flows, min refers to minimum monthly flows, and max refers to maximum monthly flows; $\log$ annual mean is the mean of the monthly flows (log) for the minimum, mean, and maximum monthly flows from Figures 1 and 2. The "Harmonic Equation Fit" column provides an estimate of how well the seasonal patterns in the different flow summaries can be summarized using a harmonic relationship. The "Correlation Summary Statistics" column provides an estimate of how the central tendency and flow extremes relate. For example, for Seneca Creek (the first entry in the table) minimum monthly flows and mean monthly flows are highly correlated suggesting that low flows are highly seasonal and follow the general pattern of the mean flows. In contrast, the maximum flows do not correlate well with the mean flows or the minimum flows, suggesting that high flows exhibit no discernible pattern or exhibit a pattern not related to the minimum or mean flows. 
Table 1 (Continued)

\begin{tabular}{|c|c|c|c|c|c|c|c|c|c|c|}
\hline $\begin{array}{l}\text { Region } \\
\text { and }\end{array}$ & $\begin{array}{c}\text { Stream } \\
\text { Name/ }\end{array}$ & Flow & & & & $\begin{array}{r}\text { Harmo } \\
\text { Equatio }\end{array}$ & $\begin{array}{l}\text { nic } \\
\text { Fit }\end{array}$ & $\begin{array}{r}\text { Correlatio } \\
\text { Statistic }\end{array}$ & $\begin{array}{l}\text { Summary } \\
s\left(r^{2} / p\right)\end{array}$ & $\begin{array}{c}\text { Log } \\
\text { Annual }\end{array}$ \\
\hline Status & Location & Summary & Phase & Amplitude & Period & p & $\underline{r^{2}}$ & Min & $\operatorname{Max}$ & Mean \\
\hline $\begin{array}{l}\text { ECST } \\
\text { PR } \\
2665\end{array}$ & $\begin{array}{l}\text { Cahaba } \\
\text { R., AL }\end{array}$ & $\begin{array}{l}\text { mean } \\
\text { min } \\
\max \end{array}$ & $\begin{array}{l}-0.17 \\
-0.21 \\
-0.16\end{array}$ & $\begin{array}{l}0.47 \\
0.36 \\
0.45\end{array}$ & $\begin{array}{l}1.92 \\
1.96 \\
2.06\end{array}$ & $\begin{array}{l}<0.001 \\
<0.001 \\
<0.001\end{array}$ & $\begin{array}{l}0.96 \\
0.88 \\
0.93\end{array}$ & $0.89 / 0.0001$ & $\begin{array}{l}0.96 / 0.0001 \\
0.83 / 0.0008\end{array}$ & $\begin{array}{l}3.075 \\
2.478 \\
3.593\end{array}$ \\
\hline $\begin{array}{l}\text { ECST } \\
\text { SR } \\
39900\end{array}$ & $\begin{array}{l}\text { Tombigbee } \\
\text { R., AL }\end{array}$ & $\begin{array}{l}\text { mean } \\
\min \\
\max \end{array}$ & $\begin{array}{l}-0.18 \\
-0.21 \\
-0.17\end{array}$ & $\begin{array}{l}0.53 \\
0.60 \\
0.38\end{array}$ & $\begin{array}{l}2.00 \\
2.08 \\
2.19\end{array}$ & $\begin{array}{l}<0.001 \\
<0.001 \\
<0.001\end{array}$ & $\begin{array}{l}0.97 \\
0.92 \\
0.92\end{array}$ & $0.90 / 0.0001$ & $\begin{array}{l}0.93 / 0.0001 \\
0.77 / 0.0033\end{array}$ & $\begin{array}{l}4.227 \\
3.455 \\
4.713\end{array}$ \\
\hline $\begin{array}{l}\text { EMNT } \\
\text { NR } \\
575\end{array}$ & $\begin{array}{l}\text { N. Fork } \\
\text { Holston } \\
\text { R., VA }\end{array}$ & $\begin{array}{l}\text { mean } \\
\text { min } \\
\text { max }\end{array}$ & $\begin{array}{l}-0.18 \\
-0.25 \\
-0.14\end{array}$ & $\begin{array}{l}0.43 \\
0.44 \\
0.32\end{array}$ & $\begin{array}{l}2.06 \\
1.99 \\
2.21\end{array}$ & $\begin{array}{l}<0.001 \\
<0.001 \\
<0.001\end{array}$ & $\begin{array}{l}0.99 \\
0.91 \\
0.86\end{array}$ & $0.82 / 0.0012$ & $\begin{array}{l}0.85 / 0.0003 \\
0.55 / 0.0658\end{array}$ & $\begin{array}{l}2.396 \\
1.784 \\
2.905\end{array}$ \\
\hline $\begin{array}{l}\text { EMNT } \\
\text { NR } \\
793\end{array}$ & $\begin{array}{l}\text { S. Yadkin } \\
\text { R., NC }\end{array}$ & $\begin{array}{l}\text { mean } \\
\min \\
\max \end{array}$ & $\begin{array}{l}-0.18 \\
-0.25 \\
-0.12\end{array}$ & $\begin{array}{l}0.19 \\
0.30 \\
0.14\end{array}$ & $\begin{array}{l}2.00 \\
2.18 \\
2.14\end{array}$ & $\begin{array}{l}<0.001 \\
<0.001 \\
<0.001\end{array}$ & $\begin{array}{l}0.92 \\
0.89 \\
0.45\end{array}$ & $0.83 / 0.0008$ & $\begin{array}{l}0.75 / 0.0046 \\
0.43 / 0.1630\end{array}$ & $\begin{array}{l}2.529 \\
2.047 \\
2.955\end{array}$ \\
\hline $\begin{array}{l}\text { EMNT } \\
\text { NR } \\
1590\end{array}$ & $\begin{array}{l}\text { Etowah } \\
\text { R., GA }\end{array}$ & $\begin{array}{l}\text { mean } \\
\min \\
\max \end{array}$ & $\begin{array}{l}-0.21 \\
-0.29 \\
-0.21\end{array}$ & $\begin{array}{l}0.27 \\
0.35 \\
0.24\end{array}$ & $\begin{array}{l}1.98 \\
2.13 \\
2.15\end{array}$ & $\begin{array}{l}<0.001 \\
<0.001 \\
<0.005\end{array}$ & $\begin{array}{l}0.97 \\
0.97 \\
0.80\end{array}$ & $0.90 / 0.0001$ & $\begin{array}{l}0.92 / 0.0001 \\
0.79 / 0.0024\end{array}$ & $\begin{array}{l}3.065 \\
2.709 \\
3.427\end{array}$ \\
\hline $\begin{array}{l}\text { EMNT } \\
\text { SR } \\
2448\end{array}$ & $\begin{array}{l}\text { French } \\
\text { Broad } \\
\text { R., NC }\end{array}$ & $\begin{array}{l}\text { mean } \\
\min \\
\max \end{array}$ & $\begin{array}{l}-0.21 \\
-0.29 \\
-0.23\end{array}$ & $\begin{array}{l}0.16 \\
0.30 \\
0.13\end{array}$ & $\begin{array}{l}2.11 \\
2.15 \\
3.34\end{array}$ & $\begin{array}{l}<0.001 \\
<0.001 \\
<0.05\end{array}$ & $\begin{array}{l}0.94 \\
0.90 \\
0.59\end{array}$ & $0.80 / 0.0016$ & $\begin{array}{l}0.52 / 0.0842 \\
0.11 / 0.7267\end{array}$ & $\begin{array}{l}3.306 \\
2.875 \\
3.648\end{array}$ \\
\hline $\begin{array}{l}\text { EMNT } \\
\text { PR } \\
8052\end{array}$ & $\begin{array}{l}\text { Potomac } \\
\text { R.; WV }\end{array}$ & $\begin{array}{l}\text { mean } \\
\text { min } \\
\max \end{array}$ & $\begin{array}{l}-0.21 \\
-0.23 \\
-0.21\end{array}$ & $\begin{array}{l}0.43 \\
0.49 \\
0.22\end{array}$ & $\begin{array}{l}2.13 \\
2.01 \\
2.37\end{array}$ & $\begin{array}{c}<0.001 \\
<0.001 \\
0.01\end{array}$ & $\begin{array}{l}0.95 \\
0.91 \\
0.70\end{array}$ & $0.92 / 0.0001$ & $\begin{array}{l}0.87 / 0.0002 \\
0.72 / 0.0080\end{array}$ & $\begin{array}{l}3.424 \\
2.789 \\
3.919\end{array}$ \\
\hline
\end{tabular}

(Sheet 2 of 5) 
Table 1 (Continued)

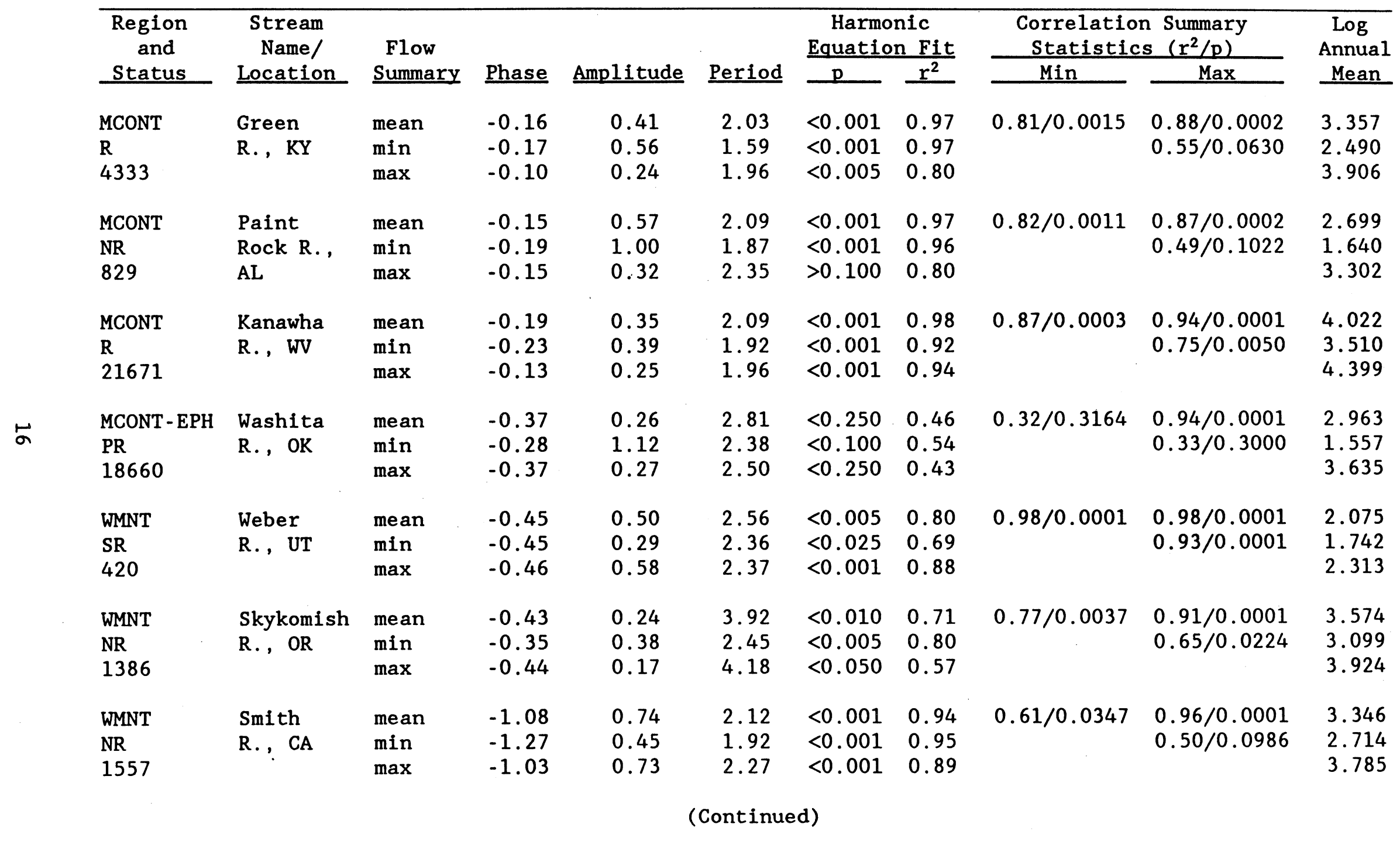


Table 1 (Continued)

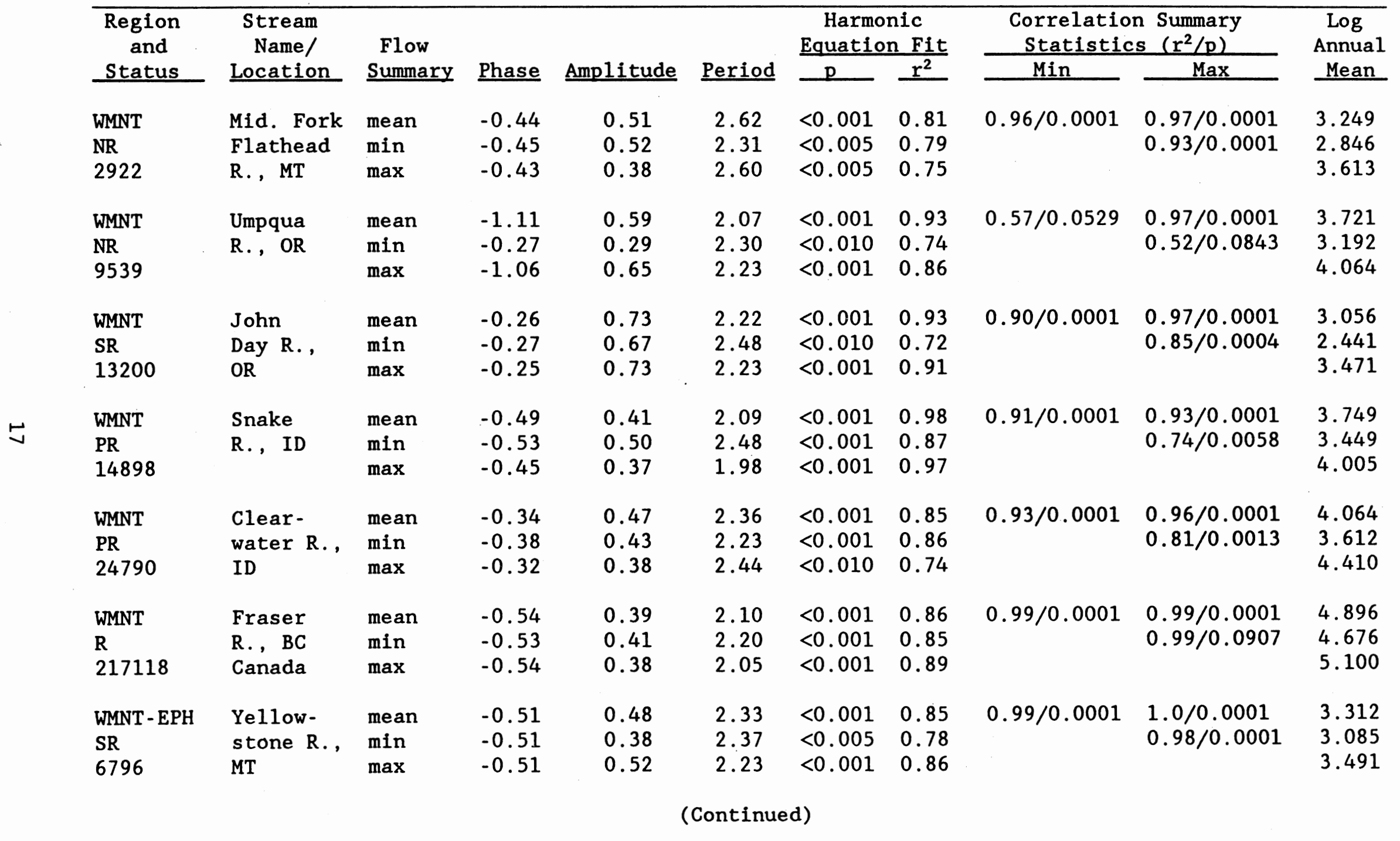

(Sheet 4 of 5) 
Table 1 (Concluded)

\begin{tabular}{|c|c|c|c|c|c|c|c|c|c|c|}
\hline \multirow{2}{*}{$\begin{array}{l}\text { Region } \\
\text { and } \\
\text { Status }\end{array}$} & \multirow{2}{*}{$\begin{array}{c}\text { Stream } \\
\text { Name/ } \\
\text { Location } \\
\end{array}$} & \multirow{2}{*}{$\begin{array}{l}\text { Flow } \\
\text { Summary }\end{array}$} & \multirow[b]{2}{*}{ Phase } & \multirow[b]{2}{*}{ Amplitude } & \multirow[b]{2}{*}{ Period } & \multicolumn{2}{|c|}{$\begin{array}{c}\text { Harmonic } \\
\text { Equation Fit }\end{array}$} & \multicolumn{2}{|c|}{$\begin{array}{l}\text { Correlation Summary } \\
\text { Statistics }\left(r^{2} / p\right)\end{array}$} & \multirow{2}{*}{$\begin{array}{c}\text { Log } \\
\text { Annual } \\
\text { Mean } \\
\end{array}$} \\
\hline & & & & & & $p$ & $\mathrm{r}^{2}$ & Min & $\operatorname{Max}$ & \\
\hline $\begin{array}{l}\text { EPH } \\
\text { SR } \\
8397\end{array}$ & $\begin{array}{l}\text { Marias } \\
\text { R., MT }\end{array}$ & $\begin{array}{l}\text { mean } \\
\min \\
\max \end{array}$ & $\begin{array}{l}-0.41 \\
-0.39 \\
-0.42\end{array}$ & $\begin{array}{l}0.48 \\
0.29 \\
0.42\end{array}$ & $\begin{array}{l}2.38 \\
2.40 \\
2.31\end{array}$ & $\begin{array}{l}<0.001 \\
<0.001 \\
<0.005\end{array}$ & $\begin{array}{l}0.84 \\
0.75 \\
0.78\end{array}$ & $0.93 / 0.0001$ & $\begin{array}{l}0.97 / 0.0001 \\
0.83 / 0.0010\end{array}$ & $\begin{array}{l}2.815 \\
2.271 \\
3.230\end{array}$ \\
\hline $\begin{array}{l}\text { EPH } \\
\text { NR } \\
12980\end{array}$ & $\begin{array}{l}\text { Humbolt } \\
\text { R., NV }\end{array}$ & $\begin{array}{l}\text { mean } \\
\min \\
\max \end{array}$ & $\begin{array}{l}-0.33 \\
-0.29 \\
-0.35\end{array}$ & $\begin{array}{l}0.71 \\
0.53 \\
0.80\end{array}$ & $\begin{array}{l}2.17 \\
2.27 \\
2.10\end{array}$ & $\begin{array}{l}<0.001 \\
<0.001 \\
<0.001\end{array}$ & $\begin{array}{l}0.80 \\
0.90 \\
0.93\end{array}$ & $0.90 / 0.0001$ & $\begin{array}{l}0.99 / 0.0001 \\
0.86 / 0.0003\end{array}$ & $\begin{array}{l}2.326 \\
1.527 \\
2.814\end{array}$ \\
\hline
\end{tabular}


and magnitude of seasonal flow patterns, particularly for seasonal high flows. Seasonality indices, especially for maximum flows, are consistently less than for eastern coastal, eastern mountain, and midcontinental stream sites (Table 2), suggesting that monthly high flows in the Western Mountain Stream Region exhibit a relatively high degree of seasonality.

13. Hydrologic patterns dominated by snowmelt and winter rain should have profound biological and evolutionary ramifications because aquatic habitat, as wetted perimeter, should be most abundant at higher streamflows. As Roff (1984) has generalized, teleost life history parameters are related; that is, age at first reproduction, natural mortality, and growth rate are determined through evolutionary coadjustments. As a group, these parameters must be at least partially determined by density independent factors typified by hydrologic variables. It seems reasonable to speculate that the intricate life history of salmonids, particularly the anadromous forms, requires an environment that is both relatively stable, persistent, and predictable. Complex life histories involving smolting, spawning runs of long distances over relatively short time periods, and prolonged incubation and rearing requirements all mediated by complex biochemical changes would be easily disrupted by hydrologic systems characterized by highly variable monthly flows, particularly during the wet season.

14. There is some evidence to suggest that atypical variability in seasonal flow patterns can have a severe impact on coldwater fishes. Erman, Andrews, and Yoder-Williams (1988) indicated that winter floods can have a severe negative effect on coldwater fishes by increased mechanical crushing of benthic fishes and eggs of fall-spawning fishes such as brook trout. Ottaway and Clarke (1981) described the downstream displacement of young trout during a short period in their development when they are particularly susceptible to entrainment. Erman (1986) hypothesized that severe droughts produced temporary local extinctions of introduced brook trout (Salvelinus fontinalis).

15. The relatively "well-behaved" hydrology (i.e., high predictability in overall flow patterns and seasonality of high flows) of western streams was a key factor in the correlated evolution of water issues and IFIM. Streams in semiarid areas having reasonably predictable hydrologic patterns are more likely to be viewed by developers as sources of firm water supply that can support agricultural or economic development. Further, these systems also support fishes whose life history is probably closely linked to the same regional hydrologic patterns that make these streams attractive sources of 
Table 2

Summary of Hydrologic Trends for Table 1 Streams by Stream Region

\begin{tabular}{|c|c|c|c|c|c|c|c|}
\hline Stream Name/Location & $\begin{array}{l}\text { Mean } \\
\text { Phase } \\
\text { Ea }\end{array}$ & $\begin{array}{l}\text { Min } \\
\text { Amp. } \\
\text { Eern C }\end{array}$ & $\begin{array}{l}\text { Max } \\
\text { Amp. }\end{array}$ & $\begin{array}{l}\text { Min- } \\
\text { Mean } \\
\text { Range } \\
\text { Regior } \\
\end{array}$ & $\begin{array}{l}\text { Max- } \\
\text { Mean } \\
\text { Range } \\
\end{array}$ & $\begin{array}{l}\text { Ratio Min } \\
\text { Amp.-Mean }\end{array}$ & $\begin{array}{l}\text { Ratio Max } \\
\text { Amp.-Mean }\end{array}$ \\
\hline $\begin{array}{l}\text { Seneca Creek, MD } \\
\text { N. Fork Edisto Cr., SC } \\
\text { Cahaba R., AL } \\
\text { Tombigbee R., AL } \\
\text { MEANS }\end{array}$ & $\begin{array}{l}-0.21 \\
-0.18 \\
-0.18 \\
-0.19 \\
-0.19\end{array}$ & $\begin{array}{l}0.35 \\
0.20 \\
0.36 \\
0.60 \\
0.38\end{array}$ & $\begin{array}{l}0.02 \\
0.03 \\
0.45 \\
0.38 \\
0.42\end{array}$ & $\begin{array}{l}0.57 \\
0.37 \\
0.60 \\
0.77 \\
0.58\end{array}$ & $\begin{array}{l}0.53 \\
0.33 \\
0.52 \\
0.49 \\
0.51\end{array}$ & $\begin{array}{l}1.63 \\
1.85 \\
1.67 \\
1.28 \\
1.61\end{array}$ & $\begin{array}{r}26.70 \\
11.10 \\
1.16 \\
1.29 \\
10.06\end{array}$ \\
\hline
\end{tabular}

Eastern Mountain Region

\begin{tabular}{|c|c|c|c|c|c|c|c|}
\hline N. Fork Holston R., VA & -0.19 & 0.44 & 0.32 & 0.61 & 0.51 & 1.39 & 1.59 \\
\hline S. Yadkin R., NC & -0.18 & 0.30 & 0.14 & 0.48 & 0.43 & 1.60 & 3.07 \\
\hline Etowah R., GA & -0.24 & 0.35 & 0.24 & 0.36 & 0.36 & 1.03 & 1.50 \\
\hline French Broad R., NC & -0.24 & 0.30 & 0.13 & 0.43 & 0.34 & 1.43 & 2.62 \\
\hline Potomac R., WV & -0.22 & $\underline{0.49}$ & $\underline{0.22}$ & $\underline{0.64}$ & $\underline{0.50}$ & 1.31 & 2.27 \\
\hline MEANS & -021 & 020 & $0 ?$ & & & & \\
\hline
\end{tabular}

Midcontinental Region

$\begin{array}{cccccccc}\text { Green R., KY } & -0.16 & 0.56 & 0.24 & 0.87 & 0.55 & 1.55 & 2.29 \\ \text { Paint Rock R., AL } & -0.16 & 1.00 & 0.32 & 1.06 & 0.60 & 1.06 & 1.88 \\ \begin{array}{c}\text { Kanawha R., WV } \\ \text { MEANS }\end{array} & \underline{-0.18} & \underline{0.39} & \underline{0.25} & \underline{0.51} & \underline{0.38} & \underline{1.31} & \underline{1.52} \\ & -0.17 & 0.65 & 0.27 & 0.81 & 0.51 & 1.31 & 1.90\end{array}$

\section{Midcontinental/Ephemeral}

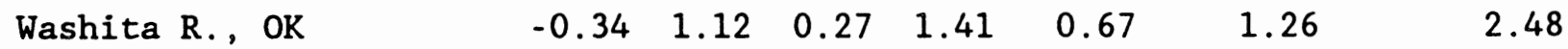

(Continued)

Note: Variables defined as follows: mean phase--mean of the phases for each flow summary; min amp.--amplitude of the monthly minimum flows; max amp.--amplitude of the monthly maximum flows; min-mean range-difference between the mean of the minimum monthly flows and the mean of the mean monthly flows (values obtained from last column in Table 1); max-mean range--difference between the mean of the maximum monthly flows and the mean of the mean monthly flows (values obtained from last column in Table 1); ratio min amp.-mean--column 4 divided by column 2 (provides estimate of the seasonal occurrence of long-term monthly low flows); ratio max amp.-mean--column 5 divided by column 3 (provides estimate of the seasonal occurrence of long-term monthly high flows). Note the consistent patterns demonstrated by western mountain streams compared with streams in other regions. 
Table 2 (Concluded)

\begin{tabular}{|c|c|c|c|c|c|c|c|}
\hline Stream Name/Location & $\begin{array}{l}\text { Mean } \\
\text { Phase }\end{array}$ & $\begin{array}{l}\text { Min } \\
\text { Amp. }\end{array}$ & $\begin{array}{l}\text { Max } \\
\text { Amp. }\end{array}$ & $\begin{array}{l}\text { Min- } \\
\text { Mean } \\
\text { Range } \\
\end{array}$ & $\begin{array}{l}\text { Max- } \\
\text { Mean } \\
\text { Range } \\
\end{array}$ & $\begin{array}{l}\text { Ratio Min } \\
\text { Amp.-Mean }\end{array}$ & $\begin{array}{l}\text { Ratio Max } \\
\text { Amp.-Mear }\end{array}$ \\
\hline \multicolumn{8}{|c|}{ Ephemeral Region } \\
\hline $\begin{array}{c}\text { Marias R., MT } \\
\text { Humbolt R., NV } \\
\text { MEANS }\end{array}$ & $\begin{array}{l}-0.41 \\
-0.32 \\
-0.37\end{array}$ & $\begin{array}{l}0.29 \\
0.53 \\
0.41\end{array}$ & $\begin{array}{l}0.42 \\
0.80 \\
0.61\end{array}$ & $\begin{array}{l}0.54 \\
0.80 \\
0.67\end{array}$ & $\begin{array}{l}0.42 \\
0.49 \\
0.46\end{array}$ & $\begin{array}{l}1.86 \\
1.51 \\
1.69\end{array}$ & $\begin{array}{l}1.00 \\
0.61 \\
0.81\end{array}$ \\
\hline \multicolumn{8}{|c|}{ Ephemeral Region/Western Mountain } \\
\hline Yellowstone R., MT & -0.51 & 0.38 & 0.52 & 0.23 & 0.18 & 0.61 & 0.35 \\
\hline \multicolumn{8}{|c|}{ Western Mountain Region } \\
\hline Weber R., UT & -0.45 & 0.29 & 0.58 & 0.33 & 0.24 & 1.14 & 0.41 \\
\hline Skykomish R., OR* & -0.41 & 0.38 & 0.17 & 0.48 & 0.35 & 1.26 & 2.06 \\
\hline Smith R., CA & -1.13 & 0.45 & 0.73 & 0.63 & 0.44 & 1.40 & 0.60 \\
\hline Md. Frk Flathead R., MT & -0.44 & 0.52 & 0.38 & 0.40 & 0.36 & 0.77 & 0.95 \\
\hline Umpqua R., OR & -0.81 & 0.29 & 0.65 & 0.53 & 0.34 & 1.83 & 0.52 \\
\hline John Day R., OR & -0.26 & 0.67 & 0.73 & 0.62 & 0.42 & 0.93 & 0.58 \\
\hline Snake R., ID & -0.48 & 0.50 & 0.37 & 0.30 & 0.26 & 0.60 & 0.70 \\
\hline Clearwater R., ID & -0.35 & 0.43 & 0.38 & 0.45 & 0.35 & 1.05 & 0.92 \\
\hline Fraser R., BC & -0.54 & $\underline{0.41}$ & $\underline{0.38}$ & $\underline{0.22}$ & $\underline{0.20}$ & $\underline{0.54}$ & $\underline{0.53}$ \\
\hline MEANS & -0.56 & 0.45 & 0.53 & 0.44 & 0.33 & 1.03 & 0.65 \\
\hline
\end{tabular}

* Not used to calculate means because hydrograph has a double period resulting from winter rain and summer snowmelt. No other Western Mountain Region stream presented had a hydrograph with two seasonal flow peaks. 
water supply. Not surprisingly, stream workers found it convenient to quantify physical habitat in terms of gaging variables and to build assessments based on areal summations of habitat for specific flow conditions--usually mean monthly flows. Impacts of water resources development on fish could then be assessed in terms that parallel standard hydrologic practices used to design reservoirs and diversion structures, facilitating analysis for both the developer and the regulator. This approach could be defensibly used to evaluate impacts on aquatic biota in streams in the Western Mountain Region for three reasons. First, the mean and range of monthly flows are highly correlated (Table 1), suggesting that mean monthly flow may be a good surrogate for hydrologic factors that limit or influence populations (whatever these factors may be). Second, the seasonality index for all three flow summaries for the Western Mountain Stream Region is relatively low, suggesting that time series approaches may capture key hydrologic behaviors of these systems.

Third, the timing of mean flows and flow extremes are predictable as evidenced by the highly significant fit of the harmonic equation to the monthly flow data (Table 1). Consequently, harmonic behavior of flows as they change more or less gradually over time can be captured by relatively long (e.g., monthly) time-steps.

\section{IFIM and Hydrology in Other Regions of the United States}

16. As Poff and Ward (1989) note, hydrologic patterns in streams in other regions of the United States may be similar to those observed in the Western Mountain Region (e.g., "mesic groundwater") or they may be dissimilar (e.g., "perennially flashy"). Depending upon the precise location of a stream, it may have hydrologic conditions similar to the streams for which IFIM was developed (e.g., Holston River, Figure 2), it may exhibit completely different hydrologic patterns (e.g., Seneca Creek, Figure 2), or it may exhibit an intermediate pattern (e.g., Humboldt River, Figure 1). The diversity of hydrologic patterns that can be found in other regions of the United States is displayed in Figure 2 and quantified in Tables 1 and 2.

17. Specific comparisons between performance of IFIM in a Western Mountain Region stream and a stream from a different stream region are not known to have been made. However, the relationship of fish population dynamics and habitat selection to hydrologic patterns described has been described for streams in or near Oklahoma (midcontinental and ephemeral stream regions). A 
review of this literature leads to four general conclusions. First, habitat selection is transitory in hydrologically variable warmwater streams with fish generally utilizing whatever habitat is available (Matthews and Hill 1980). Second, although large, unpredictable changes in the hydrograph (severe floods) in these systems do alter community composition, particularly for young-of-the-year fish (Harvey 1987), a fish community can exhibit rapid recovery to preflood structure within 8 months (Matthews 1986). Third, the degree of variability in hydrologic and water quality conditions influences community structure (Ross, Matthews, and Echelle 1985; Matthews 1987, 1988). Fourth, the habitat requirements of at least some of the fishes in this area can be described using gaging variables (mean cell velocity) supplemented by cover/substrate information (Orth and Maughn 1981, 1982). However, there is some doubt as to the value of habitat predictions based on these variables for all species (Orth and Maughn 1982), and predictions of standing crop for some species of fish are based on habitat variables not ordinarily included in IFIM studies (Layher and Maughan 1985). Indeed, Matthews (1988) suggests that depth and velocity, as normally employed in IFIM studies, may have very limited usefulness in some highly variable prairie streams. These conclusions strongly suggest that habitat selection is affected by hydrologic pattern. Highly variable hydrologic patterns seem to negate the value of habitat descriptions based on areal summations of detailed gaging measurements. Interestingly, while high flow events can occur at any season in these systems (e.g., Seneca Creek, Figure 2), the occurrence of minimum monthly flows is more predictable (Tables 1 and 2). However, this predictability is of dubious value since wetted perimeter, and hence available habitat, is at a minimum under extreme minimum flow conditions.

18. The relationship between hydrology and community structure in the other regions of the United States seems to be intermediate between the findings from the Western Mountain Region and the variable and unpredictable streams described in the above paragraph. Habitat selection by the bigmouth chub (Nocomis platyrhynchus) can be accurately described in terms of gaging variables in a Virginia stream (Lobb and Orth 1988). Mean velocity and depth explained much of the site-to-site variability in fish assemblages in South Carolina coastal plain blackwater streams (Meffe and Sheldon 1988). However, in a Missouri stream, associative responses resulted in dynamic horizontal and vertical distributions of minnows (Gorman 1988). Habitat selection by fishes appears to present a dynamic balance between available habitat, interspecific 
interactions, and predator avoidance behaviors mediated by periodic changes in flow and water quality (Gorman 1988; Gorman and Karr 1978) with young-of-theyear fishes being particularly susceptible to flow extremes (Schlosser 1985).

19. IFIM approaches may be unrealistic for evaluating impacts of river regulation on aquatic biota in those regions of the United States having flow patterns more variable than the Western Mountain Region for two reasons. First, mean monthly flows and monthly extreme flows are less correlated in streams outside the Western Mountain Region, particularly between the mean and upper flow range (Table 1). Therefore, mean flow is less likely to be a surrogate variable for other hydrologic variables because it is weakly correlated to other flow statistics that may structure the assemblages of aquatic biota in these systems. Consequently, the likelihood of serendipitously relating flow to impact on habitat using IFIM approaches is reduced. Second, seasonality indices for each flow summary are high, suggesting that time series using mean monthly flows may not be adequate to capture important habitat considerations. Measures of habitat variability may be more important in structuring these communities than flow summaries based on mean flows. Consequently, habitat time series based on mean monthly flow summaries may not correlate with abundance, recruitment, or growth of aquatic organisms, and predictions of environmental impact in terms of long-term mean flows may provide little usable information on impacts over the flow range that can be expected in these systems.

20. Seasonal high flows in some streams outside of the Western Mountain Region, particularly for coastal streams, result in regular inundation of the river flood plain. Flood plain inundation is important to the composition of stream fish assemblages (Welcomme 1989). The connection between the main channel of the stream and flood plain lakes results in an exchange of fish, and the flood plain lakes can serve as a source of recruitment (Halyk and Balon 1983). Other workers have demonstrated the importance of the flood plain both as a seasonally important habitat (Ross and Baker 1983; Kwak 1988) and as a nursery area (Copp and Penaz 1988). Interaction between the channel and off-channel areas is not ordinarily considered during the course of most IFIM studies, even though (with some innovation) it should be possible to include this factor. 


\section{IFIM and Channel Characteristics}

21. The size (particularly depth) of a stream channel has ecological and hydrologic implications on the efficacy of an IFIM analysis. In addition to ecological considerations, there are subtle interactions between gaging techniques, depth of flow, and fish size that can affect the results of an IFIM analysis. Early IFIM studies and precursor studies not only focused on western mountain streams, but further focused on streams and stream reaches of a relatively narrow size range and having relatively simple channels.

\section{IFIM and Western Mountain Stream Region Applications}

22. Brussock, Brown, and Dixon (1985), while admitting that it is difficult to make regional generalizations, notes that the Western Mountain Region has streams with several channel shapes. High gradient streams are generally described as having debris-regulated pool-riffle complexes, and midreaches are described as having poorly to moderately developed alluvial pools and riffles. Within the range of streams available in the Western Mountain Region, IFIM precursor studies and early IFIM studies were further restricted to streams best characterized as "small streams and wadeable rivers" (Stalnaker and Arnette 1976b) probably because of the interest of early stream workers in spawning and passage criteria. As listed by Stalnaker and Arnette (1976a), the spawning requirements for a number of salmonids varies between 0.2 and $3.0 \mathrm{ft}$ with a mean of $1.3 \mathrm{ft} . *$ Habitat requirements of 37 species listed in an early summary (Bovee 1974) generally fell between depths of 2 to $5 \mathrm{ft}$. Streams in this depth range are generally amenable to description and analysis using standard US Geological Survey gaging approaches for wadeable streams and rivers (Rantz et al. 1982; Herschey 1985). Channel features more difficult to portray using gaging methods such as braided channels, backwaters, sloughs, sluices, extensive numbers of snags, and flood plain connections are seldom depicted in early applications of the IFIM. Although most IFIM precursor studies were performed on stream reaches having relatively simple channel geomorphology, it is noteworthy that certain features of complex channels such as off-channel habitat for salmonids (Hartman and Brown 1987) and complex lateral habitat (backwaters) for young cutthroat

* To convert feet to meters, multiply by 0.3048 . 
trout (Oncorhynchus clarki) (Moore and Gregory 1988) are important, but these features seldom appeared in case-history studies.

23. The relative homogeneity of stream sizes may account for the early successes of IFIM in the Western Mountain Region. Streams of approximately the same order, in the same stream region, and having similar hydrologic patterns are more likely to have communities structured by the same blend of hydrologic, climatological, meteorologic, and water quality factors. Restated in terms of the River Continuum Concept, these streams are much more likely to be of the same order and, therefore, exhibit similar ecological dynamics. Consequently, obvious assumptions such as habitat limitation and more subtle assumptions such as the importance of mean cell velocity (water velocity at $6 / 10$ of the total depth) as a determining habitat variable are more likely to extend to other streams of approximately the same size within the stream region once verified for a small number of streams in a region.

24. The restricted size range and generally similar channel form of early IFIM case histories also have hydrologic implications. Techniques for gaging a river are determined by a number of factors including size, discharge, average velocity, access, and substrate. Stream gagers select stream sections for gaging that resemble the bottom of a frying pan. Velocity measurements in cells of a known cross-sectional area are used to calculate cellby-cell discharges that are, in turn, summed to estimate total discharge passing through the cross section. In simple cross sections with minimum relief, the points in the channel at which velocity readings are made become replicates of each other. In this type of channel, a gager can accurately estimate streamflow as well as determine various relationships between depth, velocity, velocity profile, and discharge. Habitat conditions within streams having simple trapezoidal channels can be either estimated directly by gaging variables, or gaging variables probably become reasonable surrogates of the hydraulic variables that determine habitat quality. The more nearly a stream channel approaches this ideal cross section (from a gaging standpoint), the more accurately a gaging-based methodology such as IFIM can be used to describe instream habitat conditions (Trihey and Wegner 1981; Rantz et al. 1982; and Herschey 1985). The many studies depicting habitat of coldwater fishes in terms of gaging variables (including nose velocity) attest to the value of this approach for some systems (e.g., Stalnaker and Arnette 1976b; Shirvell and Dungey 1983; Rimmer, Paim, and Saunders 1983; Moyle and Baltz 
1985; Bovee 1986; Cunjak and Power 1986; DeGraff and Bain 1986; Cunjak 1988; Taylor 1988).

\section{IFIM in Other Stream Regions}

25. The range of stream size, channel morphology, and regional location of streams in which IFIM could be applied is in contrast to the homogeneity of streams that characterize early applications of IFIM. For example, a large, warmwater river in the southeastern United States would be both in a different point along the river continuum from the streams and wadeable rivers used to develop IFIM and would occur in a different stream region. Outside of the Western Mountain Region, channel features such as sloughs, backwaters, numerous snags, braided channels, or seasonal connection to the flood plain provide important habitat considerations for stream organisms, particularly in coastal plain streams. For example, Benke, Van Arsdall, and Gillespie (1984) and Wallace and Benke (1984) document the importance of snag habitat for ecosystem structure and secondary production in their investigations of a subtropical southeastern river. In their system, the shifting fine sands do not support large standing crops of benthic invertebrates. The assumptions tested to validate IFIM within the Western Mountain Region may not be readily transferable to the range of streams encountered in applications of IFIM to streams in other regions.

26. The accuracy of the hydraulic programs within the PHABSIM system used to predict discharge-specific habitat varies with complexity of the channel. The hydraulic portion of an instream flow study generally can be separated into two components (Nestler, Milhous, and Layzer 1989): (a) predicting stage-discharge relationships at points along the channel profile and (b) predicting discharge-specific lateral patterns of depth and velocity at transects. Within the PHABSIM system, relatively simple, one-dimensional (in the longitudinal direction) hydraulic models and algorithms are employed to route water and to determine water surface elevations at points along the channel profile. The relatively simple algorithms used in the PHABSIM system to determine laterally or vertically varying velocities require consistent patterns between flow; depth, and velocity, either as cross-sectional averages or on a cell-by-cell basis. Complex stream channels with intricate flow patterns are difficult to simulate using the PHABSIM system because the assumption of one-dimensional flow is violated. From a hydraulic standpoint, the 
methods used in IFIM studies (as normally performed) to develop habitatdischarge relationships become increasingly less reliable as the flows deviate from those required for meeting the one-dimensional assumption.

27. The results of an IFIM analysis can also be affected by subtle interactions between size of the fish targeted for evaluation, gaging techniques, water depth, and discharge. Habitat selection by fishes is usually. described using either nose velocity (velocity at the fishes nose) or mean water column velocity with nose velocity being considered the more realistic measure. Mean velocity, the velocity usually employed in most instream flow studies, has a predictable and consistent relationship to nose velocity in streams of the size range used in the early IFIM studies. For wadeable streams, the depths at which nose velocities for salmonids are historically collected ( 0.2 to $0.4 \mathrm{ft}$ above the bottom--Table 5 in Stalnaker and Arnette (1976a)) are close to the $8 / 10$ depth of wadeable streams (i.e., if the stream is 2 to $5 \mathrm{ft}$ deep, then the distance from the $8 / 10$ depth down to the substrate is 0.4 to $1.0 \mathrm{ft}$ ). Mean velocity is routinely estimated by averaging velocity measurements at $2 / 10$ and $8 / 10$ depth. Consequently, mean water column velocity in wadeable streams may be a reasonable surrogate variable for the hydraulic variables (in this case nose velocity) that determine fish habitat selection. Additionally, velocity profiles most nearly follow the theoretical logarithmic ideal in runs and pools of simple channels having sand or gravel substrate (Walker 1988; Karim and Kennedy 1987; Chiu 1989), further strengthening the case that mean velocity and nose velocity for salmonids should be highly correlated in streams of the size range described in early IFIM studies. For deeper rivers, rivers with large substrate, or for smaller fish (in which nose velocities may be closer to the bottom), the correlation between mean water velocity and hydraulic conditions in the immediate vicinity of the fish should decrease, and mean velocity may become a less useful hydraulic variable to describe habitat selection. 


\section{PART IV: BIOLOGICAL AND LIFE HISTORY CONSIDERATIONS}

28. The biology of many coldwater biota (primarily salmonids) is well known and documented. Many studies have described salmonid habitat requirements in terms of substrate and channel flow conditions. In contrast, the biology of warmwater aquatic biota is less known and often undocumented in terms of variables used in instream flow studies. Even the term "warmwater biota" may be misleading in that it infers the existence of a distinct category of aquatic biota restricted to warmwater stream ecosystems. In reality, there may be any number of warmwater "biotic assemblages."

29. Coldwater and warmwater stream ecosystems differ substantially in species number. Small and medium-size coldwater streams are usually characterized by a relatively small number of fish species (e.g., Moyle and Vondracek 1985) and usually only several native sport fishes. It is often not difficult to identify a target species or life stage to serve as the focal point of the study. In contrast, a warmwater stream may have 30 or 40 species of fishes, with many having commercial or sport-fishing importance (e.g., Nestler et al. 1986). Consequently, it is often difficult to identify a suitable target species for the analysis. If the habitat requirements of a large number of species are evaluated, then assessment of impact is complicated because each life stage may have substantially different flow optima. Further evidence for the potential complexity of warmwater streams is provided by work relating the morphology of fish to their feeding behavior and prey selection (Keast and Webb 1966; Gatz 1981). Warmwater stream fish assemblages characteristically include families such as centrarchids and ictalurids. These families, or families having similar body forms, seldom occur in coldwater stream fish assemblages.

30. While it is impossible to directly equate a coldwater fish to its warmwater counterpart, a comparison of suitability curves for coldwater and warmwater fishes reveals that, generally, many common warmwater stream fishes are characteristically habitat generalists that can occupy a wider range of depth, velocity, and substrate conditions than coldwater biota of the same general size and shape (Figure 2). This finding coincides with the more variable hydrological patterns that many warmwater systems exhibit compared with coldwater streams and is consistent with the description of many plains fishes as "using whatever habitat is available." If suitability curves for warmwater fishes accurately depict habitat selection, then an analysis using the PHABSIM 
system for species that have broad flat suitability curves will generate habitat-discharge relationships that are broad and flat (without sharp inflection points). Such habitat-discharge relationships are difficult to use in an assessment or management context. A similar analysis performed on coldwater stream organisms, or any stream organism in which the suitability curves resemble peaks, will produce a habitat discharge relationship with a sharper inflection point.

31. The dichotomy in the form of the habitat-discharge relationship between warmwater and coldwater systems leads to one of two conclusions. First, warmwater aquatic biota may be less sensitive to changes in channel discharge than coldwater organisms. It seems a reasonable speculation that warmwater aquatic biota may respond to reduced habitat by reductions in growth or reproductive rate. Thus, within the wide ranges of depth, velocity, and cover (and perhaps water quality) in which warmwater biota occur, there may be a much narrower band for optimal growth or recruitment. Alternatively, use of mean cell water velocity, depth, and substrate/cover integrated with a monthly time-step may consistently misrepresent the underlying habitat factors that structure warmwater stream communities. True habitat factors may be masked by the alluring simplicity of gaging variables to describe habitat selection and the uncritical extension of hydrologic methods to ecological investigations. 
PART V: DISCUSSION, RECOMMENDATIONS, AND CONCLUSIONS

\section{Discussion}

32. Hydrology, as practiced by water resources developers (abstracted above the dotted line in Figure 4a), has provided the basic template upon which the quantitative features of IFIM were created and developed. Stream gaging is one of the most fundamental building blocks within the general field of surface water hydrology. In its simplest context, stream gaging involves cell-by-cell measures of conveyance area and average velocity at a transect that are summed to estimate total discharge in a stream. This information, in turn, can be used to calibrate a gage, estimate base flows, provide information to develop a hydrograph, or be used as the basis of more elaborate analyses. Stream gaging information, in conjunction with information from other gages and estimates of water need and demand, can be used to develop hydrologic summaries suitable for evaluating or selecting water resources alternatives.

33. The beauty and simplicity of IFIM, as normally practiced, is that a large part of the information necessary to make habitat assessments can be collected by slightly expanding standard stream gaging methods. The "suitability curve" concept converts depth and velocity information collected during stream gaging into estimates of habitat value, thus allowing analysis of biological impact in a framework parallel with hydrologic analysis (Figure 4a). In essence, biological assessment can be overlaid on water resources planning, and the suitability curve is a simple biological model whose form is dictated by the need to conform to simple hydrologic procedures.

34. The simplicity of application may be beguiling. Although some authors present evidence that application of IFIM to resolve water resources development conflicts is an optimal way to incorporate biological information into the decision-making process (e.g., Orth and Maughan 1981), other authors present compelling evidence that IFIM has little technical merit and, as such, cannot provide defensible input for decision makers (e.g., Mathur et al. 1984; Shirvell 1986). Verification studies (Bovee 1989) in which IFIM habitat predictions are correlated to population estimates provide equally confusing conclusions (Morhardt 1987). In some cases, there appears to be a positive correlation between habitat and biomass (e.g., Stalnaker 1979 and Loar et al. 1985) and sometimes not (e.g., Conder and Annear 1987). Both groups may be 


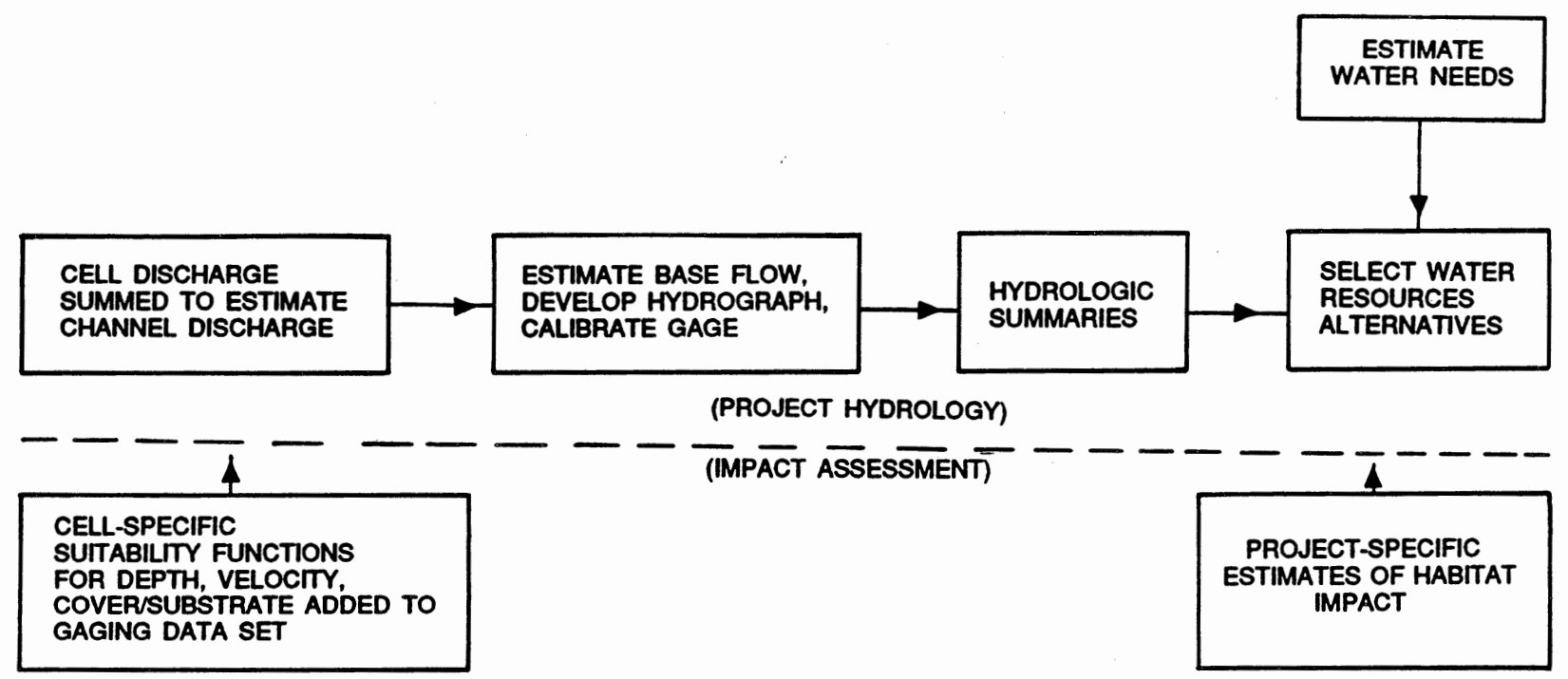

a. Conceptualization of how hydrologic analyses for project selection and impact assessment are related in IFIM studies as normally practiced
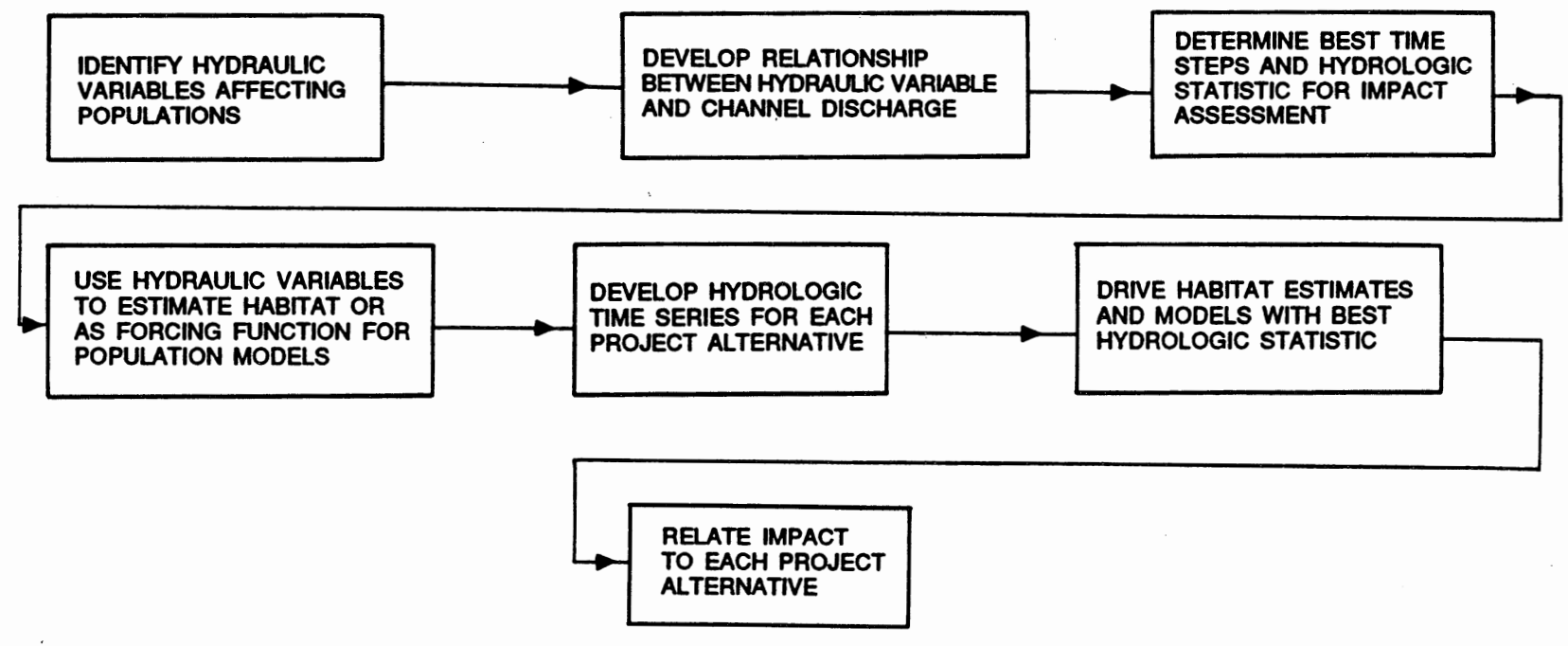

b. Recommended conceptualization of how IFIM should relate to project hydrology

Figure 4. Comparison of existing and recommended approaches for relating project impacts on aquatic biota 
correct. For streams similar in location, hydrology, and geology to the stream systems that fostered the development of IFIM, it is probably a defensible method for assessing impact of stream regulation and diversion. IFIM may be a valuable tool for addressing fish passage issues or certain salmonid life stages, such as spawning and rearing, clearly influenced by immediate hydraulic conditions. In particular, IFIM may be a valuable tool for fish populations living in streams that exhibit pronounced seasonality and predictability in flow pattern since it is in this type of stream system that the assumption of habitat limitation is most likely to be valid. However, use of the methodology is questionable in other stream systems, particularly those least similar to the stream systems that fostered the development of IFIM. In this latter case, the defensibility of the analysis may depend on a serendipitous combination of hydrology, biology, geology, channel configuration, channel size, and target life stage.

35. The inconsistency of IFIM verification studies and the conflicting conclusions of reviewers may be related to a variety of causes ranging from inadequacy of gaging variables to describe habitat selection by aquatic biota (Statzner and Higler 1986) to population limitation by factors other than aquatic habitat. The narrative presented earlier comparing stream channel characteristics, aquatic biota, and hydrologic patterns between those streams that fostered development of the IFIM with streams centered in Oklahoma suggests that habitat selection variables and factors that may limit aquatic populations may be based on hydrologic patterns. That is, aquatic biota in streams exhibiting substantially different hydrologic patterns may exhibit substantially different life history strategies that cannot be adequately depicted for assessment with a single, simple approach.

\section{Recommendations}

36. Two linked recommendations are proposed herein that will allow the IFIM to overcome its present strong regional association and develop as a national assessment methodology.

National synthesis

37. First, biologists should attempt to describe the relationship between zoogeography, regional physiography, regional hydrologic pattern (including stream order and stream size), and life history strategies of aquatic biota focusing in particular on factors that may limit or control 
population numbers. Such a national synthesis as the basis of stream investigation and description is necessary to describe how the underlying assumptions and simplifications of IFIM change from region to region and from site to site. Establishing IFIM centers across the United States does not meet this recommendation since formulation of a national synthesis is still not possible and the nagging issues of realism and applicability are not eliminated but drop from regional questions to watershed questions. A national synthesis will provide IFIM users with guiding concepts for which streamflow statistics should be related to life stage success or population dynamics (e.g., mean flow, flow ranges, flow extremes, and time-step). Second, while biological assessment should conform to a hydrologic time frame and summary variable that realistically focus on target organisms, the investigator must then relate the form of the hydrologic analysis for habitat back to the time-step and form of the analysis used for water resources planning. For example, from a water supply standpoint, a water resources engineer may be more interested in the mean monthly or lower range of streamflow, whereas an ecologist may be more interested in the amount of flood plain inundation. In this case, the ecologist must obtain the proposed operation of the water diversion structure under high flow conditions to relate the operation of the project to the potential impact on target organisms.

Microhydraulics and habitat selection

38. Instream flow studies using IFIM, as normally practiced, should not rely on unquestioned acceptance of suitability curves, since there is no compelling biological reason for many fish or aquatic macroinvertebrates, particularly benthic forms, to be directly affected by standard gaging variables. Rather, effort should be expended first to determine the hydraulic characteristics of the stream to which aquatic biota respond and then to determine how the identified characteristics relate to the gaging variables presently used in IFIM (described in Figure 4b). For example, habitat selection by salmon may be more accurately depicted by bioenergetics considerations than by suitability curves (Fausch 1983; Hughes and Dill 1990); i.e., salmon may select positions in the stream that minimize energy requirements for holding their position but that are near zones of higher velocity water likely to carry food items near the fish. Werner et al. (1983a, 1983b) have demonstrated that for warmwater fishes in lakes, optimal foraging theory can determine much of the variability in habitat selection. In summary, literature evidence suggests that fishes select habitats based on a complex interplay between risk of 
predation (discussed earlier), food availability, and bioenergetics, all mediated by flow conditions. Hydraulic variables important to the conceptualization presented by Hughes and Dill (1990), mean water velocity and depth, can be simulated by the PHABSIM system. Therefore, it seems reasonable that habitat selection of fishes should be investigated and described using more realistic approaches, such as used by Hughes and Dill (1990), that relate flow characteristics to foraging behavior, bioenergetics, or flow patterns rather than defaulting to suitability curves based solely on mean velocity, depth, and substrate.

39. IFIM could incorporate substantially more biological realism than at present if it were enriched by the ideas within the Hydraulic Stream Ecology Concept (Statzner, Gore, and Resh 1988) as recommended by Gore, Nestler, and Layzer (1990). Specifically, biologists should strive to discover and employ features of the hydraulic environment important for habitat selection rather than deferring to use of mean velocity, depth, and substrate/cover. The only constraint of this approach is that the new variable must be converted or correlated to either cell-by-cell mean velocity, transect velocity, or transect discharge so that habitat impact can be correlated to channel discharge. This latter step is important to maintain the plexus of hydrology, project planning, and impact assessment. Ultimately, as stream ecologists refine their understanding of stream ecology, IFIM could be expanded to include detailed population or community models that are partially driven by habitat considerations.

40. Options are presently available for relating fish habitat selection to stream characteristics that are more realistic than mean velocity, depth, and substrate. PHABSIM includes or could easily be modified to include the ability to evaluate shear stress, nose velocity, predation- or competitionmediated habitat refuge, feeding station behavior, or velocity diversity. Stream workers need to more frequently use some of these seldom-used options within the PHABSIM system and develop innovative approaches that could be incorporated into the PHABSIM system. Further research will surely discover new possibilities.

\section{Conclusions}

41. Implementing the above suggestions requires a change in the way many aquatic ecologists approach and interpret flowing systems. Stream 
ecologists have provided generally qualitative descriptions of stream processes that cannot be converted into rigorous hydraulic terms suitable for hydraulic simulation (Statzner, Gore, and Resh 1988). For stream ecologists to be able to contribute to the development of the next generation of IFIM, their study results must include considerably more hydrologic and hydraulic detail than currently exists (Statzner, Gore, and Resh 1988). Rather, they should follow a experimental protocol that provides a defensible description of habitat selection by target biota in their region while still maintaining the basic tenet of IFIM of assessing impact in terms roughly parallel to regional water resources planning procedures.

42. Efforts to increase the realism of IFIM investigations will also advance our understanding of aquatic ecology. Refining IFIM presents an opportunity to more rigorously and realistically describe flowing water systems in a manner consistent with habitat selection by aquatic organism and in a manner that relates habitat selection strategies with hydrologic patterns. Arguably, combining the ideas of the Hydraulic Stream Ecology Concept with concepts in IFIM, with an overview provided by regional hydrologic patterns, as described using harmonic analysis or methods similar to Poff and Ward (1990), may provide the basis for the next major advance in the ecological theory for running water systems similar to that provided by the work of Vannote et al. (1980) for the decade of the 1980s.

43. Any methodology whose development is intimately associated with a particular physiographic region must, by necessity, include in its development the constraints and biases imposed by patterns in channel geomorphology and hydrology in that region. Limitations imposed by regional considerations are not restricted to IFIM. For example, Minshall et al. (1983) points out the significance of regional factors in adjusting the River Continuum Concept (Vannote et al. 1980), and Miller et al. (1988) documents the necessity of adjusting the Index of Biotic Integrity (Karr et al. 1986) to account for regional influences.

44. As always, there is a trade-off to be made whenever an assessment methodology is modified to increase its defensibility. Assessment methodologies, similar to population models, are limited by Levin's Maxim "... population models cannot simultaneously maximize precision, generality, and realism" (Levin 1966). An assessment method such as IFIM must be general enough to be applied throughout the United States (or even in other parts of the world) and to provide predictions that are precise enough to be used as the basis of 
mitigation or operational guidelines (Gore and Nestler 1988). As IFIM is presently used, biological realism has been sacrificed to maximize generality. and precision. The thrust of the recommendations in this review is to improve realism at the expense of generality. The net results of this change are twofold. As an assessment tool, IFIM analyses will become more defensible, but also more difficult to perform and review.

45. Recent advances in IFIM appear to lead to the directions recommended in this review. In the newest release of the PHABSIM system (Milhous, Updike, and Schneider 1989), there are several programs that parallel the HABTAT program but allow evaluations beyond the use of simple gaging variables as the sole determinants of habitat quality. Researchers studying stream ecology should take advantage of the opportunities presented by these new programs not only as a means to study stream systems, but also as a means to refine and improve the programs. Additionally, the US Fish and Wildlife Service has embarked on a multiyear effort to investigate warmwater systems with the goal of adjusting and modifying IFIM for warmwater streams (Bain and Boltz 1989).

46. Water resources planning and natural resources management require an assessment methodology such as IFIM to guide decision makers. The ideas within IFIM are powerful and useful as concepts, and the basic assumption that streamflow is an important factor determining habitat quality for fishes is valid. However, the manner in which riverflow is related to habitat (or biomass) has not been generally described to the level of detail required for impact assessment for streams in all regions of the United States. Additionally, the relationship between regional hydrologic patterns and variables important to habitat selection by fishes is poorly known. Consequently, defensibility of IFIM to assess effects of river regulation or water withdrawal on stream systems outside of the Western Mountain Region is hindered until regional hydrologic and biologic factors are integrated and described in greater detail and these findings incorporated into IFIM. As Orth (1987) reiterated and Schlosser (1985) prophetically pointed out ".. understanding of stream fish assemblages will greatly profit from the development of mixed models which combine long-term studies of hydrologic regime, recruitment, and age structure with experimental approaches toward more traditional studies of foraging interactions, including competition and predation." 


\section{REFERENCES}

Allred, C. S. 1976. Data needs for decision-making, pages 401-407 in J. F. Orsborn and C. H. Allman, eds., Proceedings of the Symposium and Specialty Conference on Instream Flow Needs: Volume I, American Fisheries Society, Bethesda, MD.

Bain, M. B., and J. M. Boltz. 1989. Regulated Streamflow and Warmwater Stream Fish: A General Hypothesis and Research Agenda, US Fish and Wildlife Service Biological Report 89(18), Washington, DC 28 pp.

Bayha, K. 1976. Instream flows--the big picture, pages 95-131 in J. F. Orsborn and C. H. Allman, eds., Proceedings of the Symposium and Specialty Conference on Instream Flow Needs: Volume I, American Fisheries Society, Bethesda, MD.

Benke, A. C., T. C. Van Arsdall, Jr., and D. M. Gillespie. 1984. Invertebrate productivity in a subtropical blackwater river: the importance of habitat and life history, Ecological Monographs 54(1):25-63.

Beschta R. L., and W. S. Platts. 1986. Morphological features of small streams: significance and function, Water Resources Bulletin 22:369-379.

Bovee, K. D. 1974. The determination, assessment and design of "instream value" studies for the Northern Great Plains region, University of Montana, Final Report EPA contract No. 68-01-02413, 204 pp.

- 1982. A Guide to Stream Habitat Analysis Using the Instream Flow Incremental Methodology, US Fish and Wildlife Service Office of Biological Services, FWS/OBS-82/26, 248 pp.

1986. Development and Evaluation of Habitat Suitability Criteria for Use in the Instream Flow Incremental Methodology, US Fish and Wildlife Service Office of Biological Services, FWS/OBS-86/7, 235 pp.

1989. Everything I know about conducting IFIM validation studies, Instream Flow Chronicle, Vol 6, No. 1, available from Office of Conference Services, Colorado State University, Fort Collins, CO.

Bovee, K. D., J. A. Gore, and A. J. Silverman. 1978. Field Testing and Adaptation of a Methodology to Measure "In-Stream" Values in the Tongue River, Northern Great Plains (WGP) Region, EPA-908/4-78-004A, US Environmental Protection Agency, Washington, DC, 1978.

Bowlby J. N., and John C. Roff. 1986. Trout biomass and habitat relationships in southern Ontario streams, Transactions of the American Fisheries Society 115:503-514.

Bradley, M. D. 1976. Institutional and Policy Aspects of Instream Flow Needs, pages 79-94 in J. F. Orsborn and C. H. Allman, eds., Proceedings of the Symposium and Specialty Conference on Instream Flow Needs: Volume, I, American Fisheries Society, Bethesda, MD.

Brussock, P. P., A. V. Brown, and J. C. Dixon. 1985. Channel form and stream ecosystem models, Water Resources Bulletin 21:859-866.

Carlson, G. W. 1976. History of forest service involvement in instream flow needs, pages 295-299 in J. F. Orsborn and C. H. Allman, eds., Proceedings of the Symposium and Specialty Conference on Instream Flow Needs: Volume II, American Fisheries Society, Bethesda, MD. 
Caulfield, H. P., Jr. 1976. Perspectives on instream flow needs, pages 95131 in J. F. Orsborn and C. H. Allman, eds., Proceedings of the Symposium and Specialty Conference on Instream Flow Needs: Volume I, American Fisheries Society, Bethesda, MD.

Chiu, C. L. 1989. Velocity distribution in open channel flow, Journal of Hydraulic Engineering 115(5):576-594.

Collings, M. R. 1972. A methodology for determining flow requirements for fish in Proceedings Instream Flow Methodology Workshop, Washington Department of Ecology, Olympia, WA, pp. 72-86.

1974. Generalization of spawning and rearing discharges for several Pacific salmon species in western Washington, US Geologic Survey openfile report, $39 \mathrm{pp}$.

Collings, M. R., and G. W. Hill. 1973. The hydrology of ten streams in western Washington as related to several Pacific salmon species, US Geologic Survey Water-Resources Inv. 11-73, $149 \mathrm{pp}$.

Collings, M. R., R. W. Smith, and G. T. Higgins. 1972a. The hydrology of four streams in western Washington as related to several Pacific salmon species, Geological Survey Water-Supply Paper 1968, 109 pp.

1972b. The hydrology of four streams in western Washington as related to several Pacific salmon species, Humptulips, Elochoman, Green, and Wynoochee Rivers, US Geologic Survey open-file report, 128 pp.

Conder, A. L., and T. C. Annear. 1987. Test of weighted usable area estimates derived from a PHABSIM model for instream flow studies on trout streams, North American Journal of Fisheries Management 7:339-350.

Copp, G. H., and M. Penaz. 1988. Ecology of fish spawning and nursery zones in the flood plain, using a new sampling approach, Hydrobiologia 169:209-224.

Cunjak, R. A. 1988. Behavior and microhabitat of young Atlantic salmon (Salmo salar) during Winter, Canadian Journal of Fisheries and Aquatic Science 45:2156-2160.

Cunjak, R. A., and G. Power. 1986. Winter habitat utilization by stream resident brook trout (Salvelinus fontinalis) and brown trout (Salmo trutta), Canadian Journal of Fisheries and Aquatic Science 43:1970-1981.

Dauber, R. J. 1976. Conflicts with private and federal users, pages 132-138 in J. F. Orsborn and C. H. Allman, eds., Proceedings of the Symposium and Specialty Conference on Instream Flow Needs: Volume I, American Fisheries Society, Bethesda, MD.

DeGraff, D. A., and L. H. Bain. 1986. Habitat use by and preferences of juvenile Atlantic Salmon in two Newfoundland rivers, Transactions of the American Fisheries Society 115:671-681.

Draper, N. R., and H. Smith. 1986. Applied Regression Analysis, John Wiley and Sons, New York.

Dunham, D. K., and A. Collotzi. 1974. The transect method of stream habitat inventory, guidelines and applications, USFS, Intermountain Region, Ogden, UT, unpublished manuscript, 98 pp.

Erman, D. C. 1986. Long-term structure of fish populations in Sagehen Creek, California, Transactions of the American Fisheries Society 115:682-692. 
Erman, D. C., E. D. Andrews, and M. Yoder-Williams. 1988. Effects of winter floods on fishes in the Sierra Nevada, Canadian Journal of Fisheries and Aquatic Sciences 45:2195-2200.

Fausch, K. D. 1983. Profitable stream positions for salmonids: relating growth rate to net energy gain, Canadian Journal of Zoology 62:441-451.

Garbrecht, J., and H. W. Shen. 1988. The physical framework of the dependence between channel flow hydrographs and drainage network morphometry, Hydrological Processes 2:337-355.

Gatz, A. J. 1981. Morphologically inferred niche differentiation in stream fishes, American Midland Naturalist 106(1):10-21.

Gore, J. A., and J. M. Nestler. 1988. Opinion: instream flow studies in perspective, Regulated Rivers: Research and Management 2:93-101.

Gore, J. A., J. M. Nestler, and J. B. Layzer. 1990. Habitat Factors in Tailwaters with Emphasis on Peaking Hydropower, Technical Report EL-90-2, US Army Engineer Waterways Experiment Station, Vicksburg, MS.

Gorman, 0. T. 1988. The dynamics of habitat use in a guild of Ozark minnows, Ecological Monographs 58(1):1-18.

Gorman, 0. T., and J. R. Karr. 1978. Habitat structure and stream fish communities, Ecology 59(3):507-515.

Gurtz, M. E., G. R. Marzolf, K. T. Killingbeck, D. L. Smith, and J. V. McArthur. 1988. Hydrologic and riparian influences on the import and storage of coarse particulate organic matter in a prairie stream, Canadian Journal of Fisheries and Aquatic Sciences 45:655-665.

Halyk, L. C., and E. K. Balon. 1983. Structure and ecological production of the fish taxocene of a small floodplain system, Canadian Journal of Zoology $61: 2446-2464$.

Hartman, G. F., and T. G. Brown. 1987. Use of small, temporary, floodplain tributaries by juvenile salmonids in a west coast rain-forest drainage basin, Carnation Creek, British Columbia, Canadian Journal of Fisheries and Aquatic Sciences 44:271-281.

Harvey, B. C. 1987. Susceptibility of young-of-the-year fishes to downstream displacement by flooding, Transactions of the American Fisheries Society $116: 851-855$.

Herrington, R. B., and D. K. Dunham. 1967. A Technique for Sampling General Fish Habitat Characteristics of Streams, US Forest Service Research Paper INT-41, 12 pp.

Herschey, R. W. 1985. Streamflow Measurement, Elsevier Applied Science Publishers, New York, 553 pp.

Hobbs, B. F., E. Z. Stakhiv, and W. M. Grayman. 1989. Impact Evaluation Procedures: Theory, Practice, and Needs, Journal of Water Resources Planning and Management 115(1):2-21.

Horner, R. R., and E. B. Welch. 1981. Stream periphyton development in relation to current velocity and nutrients, Canadian Journal of Fisheries and Aquatic Sciences 38:449-457.

Hughes, J. M. R., and B. James. 1989. A hydrological regionalization of streams in Victoria, Australia with implications for stream ecology, Australian Journal of Marine and Freshwater Research 40:303-326. 
Hughes, N. F., and L. M. Dill. 1990. Position choice by drift-feeding salmonids: Model and test for arctic grayling (Thymallus arcticus) in subarctic mountain streams, interior Alaska, Canadian Journal Fisheries and Aquatic Science 47:2039-2048.

Karim, M. F., and J. F. Kennedy. 1987. Velocity and Sediment-Concentration Profiles in River Flows, Journal of Hydraulic Engineering 113(2):159-178.

Karr, J. R., K. D. Fausch, P. L. Angermeier, P. R. Yant, and I. J. Schlosser. 1986. Assessing biological integrity in running waters; a method and its rationale, Ill. Nat. Hist. Surv. Spec. Publ. 5, Urbana.

Keast, A., and D. Webb. 1966. Mouth and body form relative to feeding ecology in the fish fauna of a small lake, Lake Opinicon, Ontario, Journal of the Fisheries Research Board of Canada 23:1845-1874.

Kelley, D. W., A. J. Cordone, and G. Delisle. 1960. A method to determine the volume of flow required by trout below dams: A proposal for investigation, California Department of Fish and Game.

Kimura, D. K. 1980. Likelihood methods for the von Bertalanfffy growth curve, Fishery Bulletin 77(4):765-776.

Kwak, T. J. 1988. Lateral movement and use of floodplain habitat by fishes of the Kankakee River, Illinois, The American Midland Naturalist 120(2):241249.

Lanka, R. P., and W. A. Hubert. 1987. Relations of Geomorphology to Stream Habitat and Trout Standing Stock in Small Rocky Mountain Streams, Transactions of the American Fisheries Society 116:21-28.

Layher, W. G., and O. E. Maughan. 1985. Relations between habitat variables and catfish populations in prairie streams, Transactions of the American Fisheries Society 114:771-781.

Levin, R. 1966. The strategy of model building in population biology, American Scientist 54:421-431.

Loar, J. M., and M. J. Sale. 1981. Analysis of Environmental Issues Related to Small-scale Hydroelectric Development, Instream Flow Needs for Fishery Resources, ORNL/TM-7861, Oak Ridge National Laboratory, Oak Ridge, TN, 123 pp.

Loar, J. M., M. J. Sale, G. F. Cada, D. K. Cox, R. M. Cushman, G. K. Eddlemon, J. L. Elmore, A. J. Gatz, P. Kanciruk, J. A. Solomon, and D. S. Vaughan.

1985. Application of Habitat Evaluation Models in Southern Appalachian Trout Streams, prepared by the Oak Ridge National Laboratory, Oak Ridge, TN, ORNL/ TM-9323, 310 pp.

Lobb III, M. D., and D. J. Orth. 1988. Microhabitat use by the bigmouth chub Nocomis platyrhynchus in the New River, West Virginia, The American Midland Naturalist $120(1): 32-40$.

Mathur, D. L., Bason, W. H., Purdy, E. J., Jr., and Silver, C. A. 1984. A Critique of the Instream Flow Incremental Methodology, Canadian Journal of Fisheries and Aquatic Science 42:825-831.

Matthews, W. J. 1986. Fish faunal structure in an ozark stream: stability, persistence and a catastrophic flood, Copeia(2):388-397. 
Matthews, w. J. 1987. Physico-chemical tolerance and selectivity of stream fishes as related to their geographic ranges and local distributions, pages 111-120 in W. J. Matthews and D. C. Heins, eds., Community and Evolutionary Ecology of North American Stream Fishes, University of Oklahoma Press, Norman, OK.

1988. North American prairie streams, Journal of the North American Benthological Society 7(4):387-409.

Matthews, W. J., and L. G. Hill. 1980. Habitat partitioning in the fish community of a southwestern river, The Southwestern Naturalist 25(1):51-66.

Meffe, G. K., and A. L. Sheldon. 1988. The influence of habitat structure on fish assemblage composition in southeastern blackwater streams, The American Midland Naturalist $120(2): 225-240$.

Milhous, R. T., D. L. Wegner, and T. J. Waddle. 1981. User's Guide to the Physical Habitat Simulation System, FWS/OBS-81/43, Office of Biological Services, US Fish and Wildlife Service, Washington, DC, $475 \mathrm{pp}$.

Milhous, R. T., M. Updike, and D. Schneider. 1989. Physical Habitat Simulation System Reference Manual - Version II, Biological Report 89(16), US Fish and Wildlife Service, Washington, DC.

Miller, D. L., P. M. Leonard, R. M. Hughes, J. R. Karr, P. B. Moyle, L. H. Schrader, B. A. Thompson, R. A. Daniels, K. D. Fausch, G. A. Fitzhugh, J. R. Gammon, D. B. Halliwell, P. L. Angermeier, and D. J. Orth. 1988. Regional applications of an Index of Biotic Integrity for use in water resource management, Fisheries 13(5):12-20.

Miller, T. K. and L. J. Onesti. 1988. Interregional comparisons of alluvial stream channel morphology: great plains versus central lowlands, Water Resources Bulletin 24:1207-1217.

Minshal1, G. W., R. C. Petersen, K. W. Cummins, T. L. Bott, J. R. Sedell, C. E. Cushing, and R. L. Vannote. 1983. Interbiome comparisons of stream ecosystem dynamics, Ecological Monographs 53(1):1-25.

Moore, K. M. S., and S. V. Gregory. 1988. Response of young-of-the-year trout to manipulation of habitat structure in a small stream, Transactions of the American Fisheries Society 117:162-170.

Morhardt, J. E. 1987. Instream Flow Methodologies, prepared by EA Engineering, Science and Technology, Inc., 41A Lafayette Circle, Lafayette, California, 94549 for The Electrical Power Research Institute, 3412 Hillyview Avenue, Palo Alto, CA.

Moyle, P. B., and D. M. Baltz. 1985. Microhabitat use by an assemblage of California stream fishes: developing criteria for instream flow determinations, Transaction of the American Fisheries Society 114:695-704.

Moyle, P. B., and B. Vondracek. 1985. Persistence and structure of the fish assemblage in a small California stream, Ecology 66(1):1-13.

Munther, G. L. 1975. Fishery effects of irrigation diversion and related structures--An interim fisheries management plan for the Sawtooth National Recreation Area, USFS, Region 4, Sawtooth National Forest, 119 pp.

Nestler, J. M., J. Fritschen, R. T. Milhous, and J. Troxel. 1986. Effects of Flow Alterations on Trout, Angling, and Recreation in the Chat tahoochee River between Buford Dam and Peachtree Creek, Technical Report E-86-10, US Army Engineer Waterways Experiment Station, Vicksburg, MS, 102 pp. 
Nestler, J. M., R. T. Milhous, and J. B. Layzer. 1989. Instream Habitat Modeling Techniques, in Alternatives in Regulated River Management, J. A. Gore and G. E. Petts, eds., CRC Press Inc., Boca Raton, FL, pp 295-315.

Orth, D. J. 1987. Ecological considerations in the development and application of instream flow-habitat models, Regulated Rivers: Research and Management 1:171-181.

Orth, D. J., and O. E. Maughan. 1981. Estimated stream flow requirements for fishes of the Washita River below Foss Reservoir, Western Oklahoma, Water Resources Bulletin 17(5):831-843.

- 1982. Evaluation of the Incremental Methodololgy for Recommending Instream Flows for Fishes, Trans. Am. Fish. Soc. 111:413-445.

Ottaway, E. M., and A. Clarke. 1981. A preliminary investigation into the vulnerability of young trout (Salmo trutta L.) and Atlantic salmon ( $S$. salar L.) to downstream displacement by high water velocities, Journal of Fish Biology 19:135-145.

Patten, B. D. 1979. Summary Report of Module B - Instream Fishery Ecosystems, Instream Flow Criteria and Modeling Workshop, Colorado State University, Fort Collins, Colorado, Experiment Station, Is No 40.

Petts, G. E. 1984. Impounded Rivers: Perspectives for Ecological Management, John Wiley and Sons, New York, 326 pp.

Poff, N. L., and J. V. Ward. 1989. Implications of streamflow variability and predictability for lotic community structure: a regional analysis of streamflow patterns, Canadian Journal of Fisheries and Aquatic Science $46: 1805-1818$.

1990. The physical template of lotic systems: recovery in the context of historical patterns of spatio-temporal heterogeneity, Environmental Management (in press).

Pruitt, T. A., and R. L. Nadeau. 1978. Recommended stream resource maintenance flows on seven southern Idaho streams, US Fish and Wildlife Service Office of Biological Services, FWS/OBS-78/68, 60 pp.

Rantz, S. E. and others. 1982. Measurement and Computation of Streamflow: Volume 1, Measurement of Stage and Discharge, US Geological Survey WaterSupply Paper 2175.

Reiser, D. W., T. A. Wesche, and C. Estes. 1989. Status of Instream Flow Legislation and Practices in North America, Fisheries. Vol 14, No. 2, pp 22-29.

Rimmer, D. M., U. Paim, and R. L. Saunders. 1983. Changes in the selection of microhabitat by juvenile Atlantic Salmon (Salmo salar) at the summer-autumn transition in a small river, Canadian Journal of Fisheries and Aquatic Sciences 41:469-475.

Roff, D. A. 1984. The evolution of life history parameters in teleosts, Canadian Journal of Fisheries and Aquatic Science 41:989-1000

Ross, S. T., and J. A. Baker. 1983. The response of fishes to periodic spring floods in a southeastern stream, The American Midland Naturalist $109(1): 1-14$. 
Ross, S. T., W. J. Matthews, and A. A. Echelle. 1985. Persistence of stream fish assemblages: effects of environmental change, The American Naturalist $126(1): 24-40$

Sams, R. E., and L. S. Pearson. 1963. A study to develop methods for determining spawning flows for anadromous salmonids, Oregon Fish Comm., unpublished manuscript, $56 \mathrm{pp}$.

SAS Institute Inc. 1988. SAS Language Guide for Personal Computers, Release 6.03 Edition, SAS Institute Inc., Cary, NC, 558 pp.

Schlosser, I. J. 1985. Flow regime, juvenile abundance, and the assemblage structure of stream fishes, Ecology 66(5):1484-1490.

Sedell, J. R., R. J. Naiman, K. W. Cummins, G. W. Minshall, and R. L. Vannote. 1978. Transport of particulate organic matter in streams as a function of physical processes, Verh. Internat. Verein. Limnol., pp. 1366-1375.

Shirvell, C. S. 1986. Pitfalls of Physical Habitat Simulation in the Instream Flow Incremental Methodology, Canadian Technical Report of Fisheries and Aquatic Sciences No. 1460, pp 1-68.

Shirvel1, C. S., and R. G. Dungey. 1983. Microhabitats chosen by Brown Trout for Feeding and Spawning in Rivers, Transactions of the American Fisheries Society 112:355-367.

Stalnaker, C. B. 1979. Use of habitat structure preferenda in flow regimes, in The Ecology of Regulated Streams, J. V. Ward and J. A. Stanford, eds., Plenum Press, New York, 398 pp.

Stalnaker, C. B., and J. L. Arnette. 1976a. Methodologies for determining instream flows for fish and other aquatic life, in Stalnaker, C. B. and J. L. Arnette, eds., Methodologies for the Determination of Stream Resource Flow Requirements: An Assessment, prepared for US Fish and Wildlife Service, Office of Biological Services, Western Water Allocation by Utah State University, Logan, UT, pp 89-138.

1976b. Methodologies for the Determination of Stream Resource Flow Requirements: An Assessment, prepared for US Fish and Wildlife Service, Office of Biological Services, Western Water Allocation by Utah State University, Logan, UT, 199 pp.

Statzner B., and B. Higler. 1985. Questions and comments on the river continuum concept, Canadian Journal of Fisheries and Aquatic Sciences 42:1038-1044.

- 1986. Stream Hydraulics as a Major Determinant of Benthic Invertebrate Zonation, Freshwater Biology 16:127-139.

Statzner, B., J. A. Gore, and V. H. Resh. 1988. Hydraulic Stream Ecology: Observed Patterns and Potential Application, North American Benthological Society 7:307-360.

Taylor, E. B. 1988. Water temperature and velocity as determinants of microhabitats of juvenile chinook and coho salmon in a laboratory stream channel, Transactions of the American Fisheries Society 117:22-28.

Thompson, K. E. 1972. Determining streamflows for fish life, in Proceedings Instream Flow Requirement Workshop, Pacific N.W. River Basins Comm., Portland, OR, pp 31-50. 
Trihey, E. W., and D. L. Wegner. 1981. Field data collection procedures for use with the physical habitat simulation system of the Instream Flow Group, USDI Fish and Wildlife Service, Cooperative Instream Flow Group, Fort Collins, CO, 151 pp.

US Geological Survey. 1988. Streamflow during March, published by the US Department of the Interior, Geological Survey, 419 National Center, Reston VA 22092, 19 pp.

Vannote, R. L., G. W. Minshall, K. W. Cummins, J. R. Sedell, and C. E. Cushing. 1980. The river continuum concept, Canadian Journal of Fisheries and Aquatic Science 37:130-137.

Walker, J. F. 1988. General two-point method for determining velocity in open channel, Journal of Hydraulic Engineering 114(7):801-805.

Wallace, J. B., and A. C. Benke. 1984. Quantification of wood habitat in subtropical coastal plain streams, Canadian Journal of Fisheries and Aquatic Sciences 41:1643-1652.

Waters, B. F. 1976. A methodology for evaluating the effects of different streamflows on salmonid habitat, pages 254-266 in J. F. Orsborn and C. H. Allman, eds., Proceedings of the Symposium and Specialty Conference on Instream Flow Needs: Volume II, American Fisheries Society, Bethesda, MD.

Welcomme, R. 1989. Floodplain fisheries management, in Alternatives in Regulated River Management, J. A. Gore and G. E. Petts, eds., CRC Press Inc., Boca Raton, FL, pp 209-233.

Werner, E. E., G. G. Mittelbach, D. J. Hall, and J. F. Gilliam. 1983b. Experimental tests of optimal habitat use in fish: the role of habitat profitability, Ecology 64(6):1525-1539.

Werner, E. E., J. F. Gilliam, D. J. Hall, and G. G. Mittelbach. 1983a. An experimental test of the effects of predation risk on habitat use in fish, Ecology 64(6):1540-1548. 

Public reporting burden for this collection of information is estimated to average 1 hour per response, including the time for reviewing instructions, searching existing data sources. gathering and maintaining the data needed, and completing and reviewing the collection of information. Send comments regarding this burden estimate or any other aspect of this collection of information, including suggestions for reducing this burden. to Washington Headquarters Services. Directorate for intormation Operations and Reports. 3215 Jefferson Davis Highway, Suite 1204, Arlington, VA 22202-4302, and to the Office of Management and Budget. Paperwork Reduction Project (0704-0188), Washington, DC 20503.

\begin{tabular}{|l|l|l}
\hline 1. AGENCY USE ONLY (Leave blank) & $\begin{array}{c}\text { 2. REPORT DATE } \\
\text { January } 1993\end{array}$ & $\begin{array}{c}\text { 3. REPORT TYPE AND DATES COVERED } \\
\text { Final report }\end{array}$
\end{tabular}

4. ITLE AND SUBPILE Incremental Methodology: A Synopsis with Recommendations for Use and Suggestions for

Future Research

6. AUTHOR(S)

John M. Nestler

\section{PERFORMING ORGANIZATION NAME(S) AND ADDRESS(ES)}

US Army Engineer Waterways Experiment Station

Environmental Laboratory

3909 Halls Ferry Road

Vicksburg, MS 39180-6199

9. SPONSORING/MONITORING AGENCY NAME(S) AND ADDRESS(ES)

US Army Corps of Engineers

Washington, DC 20314-1000
5. FUNDING NUMBERS

WU 32494

\section{SUPPLEMENTARY NOTES}

Available from National Technical Information Service, 5285 Port Royal Road, Springfield, VA 22161

\section{2a. DISTRIBUTION / AVAILABILITY STATEMENT}

Approved for public release; distribution

is unlimited

\section{ABSTRACT (Maximum 200 words)}

An overview of the Instream Flow Incremental Methodology (IFIM) is presented with particular emphasis on guiding influences of western hydrology, regional geology, important pre-IFIM studies, and select biological considerations. These influences are compared and contrasted with those prevalent in other regions of the United States. These influences are used to partially explain conflicting literature evaluations of IFIM and also to serve as the basis of suggestions for improving the methodology, particularly for eastern and southeastern United States applications. Improving IFIM by incorporating tenets of the Hydraulic Stream Ecology Concept and thus relaxing the present tight linkage between hydrology and biology characterizing many applications of the methodology is suggested. This suggestion enhances the realism and defensibility of the methodology for assessment and also makes IFIM an investigative tool for advancing stream ecology.

\begin{tabular}{|c|c|c|c|}
\hline $\begin{array}{l}\text { 14. SUBJECT TERMS } \\
\text { Environmental assessment } \\
\text { Instream flow incremental } \\
\text { methodology }\end{array}$ & \multicolumn{2}{|c|}{$\begin{array}{l}\text { Physical habitat simulation } \\
\text { Regional considerations } \\
\text { Warmwater fishes }\end{array}$} & $\begin{array}{l}\text { 15. NUMBER OF PAGES } \\
48 \\
\text { 16. PRICE CODE }\end{array}$ \\
\hline $\begin{array}{l}\text { 17. SECURITY CLASSIFICATION } \\
\text { OF REPQRL } \\
\text { UNCLASSIED }\end{array}$ & $\begin{array}{l}\text { 18. SECURITY CLASSIFICATION } \\
\text { OF THIS PAGE SASIFIED }\end{array}$ & $\begin{array}{l}\text { 19. SECURITY CLASSIFICATION } \\
\text { OF ABSTRACT }\end{array}$ & 20. LIMITATION OF ABSTRACT \\
\hline
\end{tabular}


